\title{
A Time- and Message-Optimal Distributed Algorithm for Minimum Spanning Trees
}

\author{
GOPAL PANDURANGAN, University of Houston \\ PETER ROBINSON, City University of Hong Kong \\ MICHELE SCQUIZZATO, University of Padova
}

\begin{abstract}
This article presents a randomized (Las Vegas) distributed algorithm that constructs a minimum spanning tree (MST) in weighted networks with optimal (up to polylogarithmic factors) time and message complexity. This algorithm runs in $\tilde{O}(D+\sqrt{n})$ time and exchanges $\tilde{O}(m)$ messages (both with high probability), where $n$ is the number of nodes of the network, $D$ is the hop-diameter, and $m$ is the number of edges. This is the first distributed MST algorithm that matches simultaneously the time lower bound of $\tilde{\Omega}(D+\sqrt{n})[10]$ and the message lower bound of $\Omega(m)$ [31], which both apply to randomized Monte Carlo algorithms.

The prior time and message lower bounds are derived using two completely different graph constructions; the existing lower-bound construction that shows one lower bound does not work for the other. To complement our algorithm, we present a new lower-bound graph construction for which any distributed MST algorithm requires both $\tilde{\Omega}(D+\sqrt{n})$ rounds and $\Omega(m)$ messages.
\end{abstract}

\section{CCS Concepts: • Theory of computation $\rightarrow$ Distributed algorithms;}

Additional Key Words and Phrases: Distributed algorithms, minimum spanning trees

\section{ACM Reference format:}

Gopal Pandurangan, Peter Robinson, and Michele Scquizzato. 2019. A Time- and Message-Optimal Distributed Algorithm for Minimum Spanning Trees. ACM Trans. Algorithms 16, 1, Article 13 (November 2019), 27 pages.

https://doi.org/10.1145/3365005

\section{INTRODUCTION}

The minimum-weight spanning tree (MST) construction problem is one of the central and most studied problems in distributed computing [43]. A long line of research aimed at developing efficient distributed algorithms for the MST problem started more than thirty years ago with the

A preliminary version of this paper [42] appeared in the Proceedings of the 49th Annual ACM Symposium on the Theory of Computing (STOC 2017). G. Pandurangan was supported, in part, by NSF grants CCF-1527867, CCF-1540512, IIS-1633720, and CCF-1717075, and by US-Israel Binational Science Foundation (BSF) grant 2016419. Most of this work was done while M. Scquizzato was at the University of Houston supported, in part, by NSF grants CCF-1527867, CCF-1540512, and IIS1633720, and at KTH Royal Institute of Technology supported, in part, by the European Research Council (ERC) under the European Union's Horizon 2020 research and innovation programme under grant agreement No 715672.

Authors' addresses: G. Pandurangan, Department of Computer Science, University of Houston, 3551 Cullen Blvd, Houston, TX 77204, USA; email: gopalpandurangan@gmail.com; P. Robinson, Department of Computer Science, City University of Hong Kong, 83 Tat Chee Avenue, Kowloon, Hong Kong; email: peter.robinson@cityu.edu.hk; M. Scquizzato, Department of Mathematics, University of Padova, Via Trieste 63, 35121 Padova, Italy; email: scquizza@math.unipd.it.

Permission to make digital or hard copies of all or part of this work for personal or classroom use is granted without fee provided that copies are not made or distributed for profit or commercial advantage and that copies bear this notice and the full citation on the first page. Copyrights for components of this work owned by others than ACM must be honored. Abstracting with credit is permitted. To copy otherwise, or republish, to post on servers or to redistribute to lists, requires prior specific permission and/or a fee. Request permissions from permissions@acm.org.

(c) 2019 Association for Computing Machinery.

1549-6325/2019/11-ART13 \$15.00

https://doi.org/10.1145/3365005 
seminal article of Gallager et al. [14], which presented a distributed algorithm that constructs an MST in $O(n \log n)$ rounds exchanging a total of $O(m+n \log n)$ messages, where $n$ and $m$ denote the number of nodes and the number of edges of the network, respectively. ${ }^{1}$ The message complexity of this algorithm is (essentially) optimal [31], but its time complexity is not. Hence, further research concentrated on improving the time complexity. The time complexity was first improved to $O(n \log \log n)$ by Chin and Ting [5], further improved to $O\left(n \log ^{*} n\right)$ by Gafni [13], and then to $O(n)$ by Awerbuch [2] (see also [12]). The $O(n)$ bound is existentially optimal in the sense that there exist graphs for which this is the best possible.

This was the state of the art till the mid-nineties when Garay et al. [15] raised the question of whether it is possible to identify graph parameters that can better capture the complexity of distributed network computations. In fact, for many existing networks, their hop-diameter $D$ is significantly smaller than the number of vertices $n$, and therefore it is desirable to design protocols whose running time is bounded in terms of $D$ rather than in terms of $n$. Garay et al. [15] gave the first such distributed algorithm for the MST problem with running time $O\left(D+n^{0.614} \log ^{*} n\right)$, which was later improved by Kutten and Peleg [32] to $O\left(D+\sqrt{n} \log ^{*} n\right) .^{2}$ However, both these algorithms are not message-optimal, ${ }^{3}$ as they exchange $O\left(m+n^{1.614}\right)$ and $O\left(m+n^{1.5}\right)$ messages, respectively. All the above results, as well as the one in this article, hold in the synchronous CONGEST model of distributed computing, a well-studied standard model of distributed computing [45] (see Section 1.1).

The lack of progress in improving the result of [32], and in particular breaking the $\tilde{O}(\sqrt{n})$ barrier, ${ }^{4}$ led to work on lower bounds for the distributed MST problem. Peleg and Rubinovich [46] showed that $\Omega(D+\sqrt{n} / \log n)$ time is required by any distributed algorithm for constructing an MST, even on networks of small diameter $(D=\Omega(\log n))$; thus, this result establishes the asymptotic near-tight optimality of the algorithm of [32]. The lower bound of Peleg and Rubinovich applies to exact, deterministic algorithms. Later, the lower bound was improved to $\Omega(D+\sqrt{n / \log n})$ and was shown for randomized (Monte Carlo) and approximation algorithms as well [7, 10].

To summarize, the state of the art for distributed MST algorithms is that there exist algorithms which are either time-optimal (i.e., they run in $\tilde{O}(D+\sqrt{n})$ time) or message-optimal (i.e., they exchange $\tilde{O}(m)$ messages), but not simultaneously both. Indeed, the time-optimal algorithms of [9, 32] (as well as the sublinear time algorithm of [15]) are not message-optimal, i.e., they require asymptotically much more than $\Theta(m)$ messages. In contrast, the known message-optimal algorithms for MST (in particular, $[2,14]$ ) are not time-optimal, i.e., they take significantly more time than $\tilde{O}(D+\sqrt{n})$. In their 2000 SICOMP paper [46], Peleg and Rubinovich raised the question of whether one can design a distributed MST algorithm that is simultaneously optimal with respect to time and message complexity. In 2011, Kor et al. [28] also raised this question and showed that distributed verification of MST, i.e., verifying whether a given spanning tree is MST or not, can be done in optimal messages and time, i.e., there exists a distributed verification algorithm that uses $\tilde{O}(m)$ messages and runs in $\tilde{O}(D+\sqrt{n})$ time, and that these are optimal bounds for MST verification. However, the original question for MST construction remained open.

The above question addresses a fundamental aspect in distributed algorithms, namely the relationship between the two basic complexity measures of time and messages. The simultaneous optimization of both time and message complexity has been elusive for several fundamental

\footnotetext{
${ }^{1}$ The original algorithm has a message complexity of $O(m \log n)$, but it can be improved to $O(m+n \log n)$.

${ }^{2}$ The $\log ^{*} n$ factor can, in all respective algorithms, be reduced to $\sqrt{\log ^{*} n}$, by growing components to a size larger by a factor $\sqrt{\log ^{*} n}$ in the respective first phase.

${ }^{3}$ In this article, henceforth, when we say "optimal" we mean "optimal up to a polylog $(n)$ factor".

${ }^{4} \tilde{O}(f(n))$ and $\tilde{\Omega}(f(n))$ denote $O(f(n) \cdot \operatorname{polylog}(f(n)))$ and $\Omega(f(n) / \operatorname{polylog}(f(n)))$, respectively.
} 
problems (including MST, shortest paths, and random walks), and consequently research in the last three decades in distributed algorithms has focused mainly on optimizing either one of the two measures separately. However, in various modern and emerging applications such as resourceconstrained communication networks and distributed computation of large-scale data, it is crucial to design distributed algorithms that optimize both measures simultaneously [23, 27].

\subsection{Model and Definitions}

We first briefly describe the distributed computing model in which our algorithm (as well as all the previously discussed MST algorithms $[2,5,9,13-15,32])$ is specified and analyzed. This is the CONGEST model (see, e.g., [45]), which is now standard in the distributed computing literature.

A point-to-point communication network is modeled as an undirected weighted graph $G=$ $(V, E, w)$, where the vertices of $V$ represent the processors, the edges of $E$ represent the communication links between them, and $w(e)$ is the weight of edge $e \in E$. Without loss of generality, we assume that $G$ is connected. $D$ denotes the hop-diameter (that is, the unweighted diameter) of $G$, and in this paper by diameter we always mean hop-diameter. We also assume that the weights of the edges of the graph are all distinct. This implies that the MST of the graph is unique. The definitions and the results generalize readily to the case where the weights are not necessarily distinct. Each node hosts a processor with limited initial knowledge. Specifically, we make the common assumption that each node has unique identity numbers (this is not essential, but simplifies presentation), and at the beginning of computation each vertex $v$ accepts as input its own identity number and the weights of the edges incident to it. Thus, a node has only local knowledge. Specifically, we assume that each node has ports (each port having a unique port number); each incident edge is connected to one distinct port. A node does not have any initial knowledge of the other endpoint of its incident edge (which node it is connected to or the port number that it is connected to). This model is referred to as the clean network model in [45] and is also sometimes referred to as the $K T_{0}$ model, i.e., the initial (K)nowledge of all nodes is restricted (T)ill radius 0 (i.e., just the local knowledge) [45]. The $K T_{0}$ model is a standard model in distributed computing and typically used in the literature (see e.g., [1,35, 45, 49]), including all the prior results on distributed MST (e.g., [2, 5, 14, 15, 32]) with a notable exception ([26], discussed in detail in Section 1.3).

The vertices are allowed to communicate through the edges of the graph $G$. It is assumed that communication is synchronous and occurs in discrete rounds (time steps). In each time step, each node $v$ can send an arbitrary message of $O(\log n)$ bits through each edge $e=(v, u)$ incident to $v$, and each message arrives at $u$ by the end of this time step. (If unbounded-size messages are allowed-this is the so-called LOCAL model-the MST problem can be trivially solved in $O(D)$ time [45].) The weights of the edges are at most polynomial in the number of vertices $n$, and therefore the weight of a single edge can be communicated in one time step. This model of distributed computation is called the CONGEST $(\log n)$ model or simply the CONGEST model [45]. We also assume that each vertex has access to the outcome of unbiased private coin flips.

The efficiency of distributed algorithms is traditionally measured by their time and message (or, communication) complexities. Time complexity measures the number of synchronous rounds taken by the algorithm, whereas message complexity measures the total amount of messages sent and received by all the processors during the execution of the algorithm. Both complexity measures crucially influence the performance of a distributed algorithm. We say that a problem enjoys singular optimality if it admits a distributed algorithm whose time and message complexity are both optimal. When the problem fails to admit such a solution, namely, algorithms with better time complexity for it necessarily incur higher message complexity and vice versa, we say that the problem exhibits a time-message tradeoff. 
Table 1. Summary of Upper Bounds on the Complexity of Distributed MST

\begin{tabular}{lcc}
\hline Reference & Time Complexity & Message Complexity \\
\hline Gallager et al. [14] & $O(n \log n)$ & $O(m+n \log n)$ \\
Awerbuch [2] & $O(n)$ & $O(m+n \log n)$ \\
Garay et al. [15] & $O\left(D+n^{0.614} \log ^{*} n\right)$ & $O\left(m+n^{1.614}\right)$ \\
Kutten and Peleg [32] & $O\left(D+\sqrt{n} \log ^{*} n\right)$ & $O\left(m+n^{1.5}\right)$ \\
Elkin [9] & $\tilde{O}(\mu(G, w)+\sqrt{n})^{\mathrm{a}}$ & $O\left(m+n^{1.5}\right)$ \\
This article & $\tilde{O}(D+\sqrt{n})$ & $\tilde{O}(m)$ \\
\hline
\end{tabular}

aarameter $\mu(G, w)$ is called MST-radius-see Section 1.3.

\subsection{Our Results}

Distributed MST Algorithm. In this article, we present a distributed MST algorithm in the CONGEST model which is simultaneously time- and message-optimal. The algorithm is randomized Las Vegas, and always returns the MST. The running time of the algorithm is $\tilde{O}(D+\sqrt{n})$ and the message complexity is $\tilde{O}(m)$, and both bounds hold with high probability. ${ }^{5}$ This is the first distributed MST algorithm that matches simultaneously the time lower bound of $\tilde{\Omega}(D+\sqrt{n})[7,10]$ and the message lower bound of $\Omega(m)$ [31], which both apply even to randomized Monte Carlo algorithms. In terms of the terminology introduced earlier, we can therefore say that the distributed MST problem exhibits singular optimality up to polylogarithmic factors. Table 1 summarizes the known upper bounds on the complexity of distributed MST.

Lower Bound. Both the aforementioned time and message lower bounds are existential, and are derived using two completely different graph constructions. However, the graph used to show one lower bound does not work for the other. To complement our main result, in Section 4 we present a new graph construction for which any distributed MST algorithm requires both $\tilde{\Omega}(D+\sqrt{n})$ rounds and $\Omega(m)$ messages.

\subsection{Other Related Work}

Given the importance of the distributed MST problem, there has been significant work over the last 30 years on this problem and related aspects. Besides the prior work already mentioned in Section 1, we now discuss other relevant work on distributed MST. Additional details can be found in a recent survey on the problem [43].

Other Distributed MST Algorithms. Elkin [9] showed that a parameter called MST-radius captures the complexity of distributed MST algorithms better. The MST-radius, denoted by $\mu(G, w)$, and which is a function of the graph topology as well as the edge weights, roughly speaking is the maximum radius each vertex has to examine to check whether any of its edges is in the MST. Elkin devised a distributed protocol that constructs the MST in $\tilde{O}(\mu(G, w)+\sqrt{n})$ time. The ratio between diameter and MST-radius can be as large as $\Theta(n)$, and consequently, on some inputs, this protocol is faster than the protocol of [32] by a factor of $\Omega(\sqrt{n})$. However, a drawback of this protocol (unlike the previous MST protocols [5, 13-15, 32]) is that it cannot detect the termination of the algorithm in that time (unless $\mu(G, w)$ is given as part of the input). On the other hand, it can be shown that for distributed MST algorithms that correctly terminate $\Omega(D)$ is a lower bound on the running time [30,46]. (In fact, Kutten et al. [30] shows that, for every sufficiently large $n$ and every function $D(n)$ with $2 \leq D(n)<n / 4$, there exists a graph $G$ of $n^{\prime} \in \Theta(n)$ nodes and diameter

\footnotetext{
${ }^{5}$ Throughout, with high probability (w.h.p.) means with probability $\geq 1-1 / n^{\Omega(1)}$, where $n$ is the network size.
} 
$D^{\prime} \in \Theta(D(n))$ which requires $\Omega\left(D^{\prime}\right)$ rounds to compute a spanning tree with constant probability.) We also note that the message complexity of Elkin's algorithm is $O\left(m+n^{1.5}\right)$.

Some classes of graphs admit efficient MST algorithms that beat the general $\tilde{\Omega}(D+\sqrt{n})$ time lower bound. This is the case for planar graphs, graphs of bounded genus, treewidth, or pathwidth [16, 21, 22], and graphs with small random walk mixing time [18].

Time Complexity. From a practical perspective, given that MST construction can take as much as $\Omega(\sqrt{n / \log n})$ time even in low-diameter networks, it is worth investigating whether one can design distributed algorithms that run faster and output an approximate minimum spanning tree. The question of devising faster approximation algorithms for MST was raised in [46]. Elkin [10] later established a hardness result on distributed MST approximation, showing that approximating the MST problem on a certain family of graphs of small diameter within a ratio $H$ requires essentially $\Omega(\sqrt{n / H \log n})$ time. Khan and Pandurangan [25] showed that there can be an exponential time gap between exact and approximate MST construction by showing that there exist graphs where any distributed (exact) MST algorithm takes $\Omega(\sqrt{n} / \log n)$ rounds, whereas an $O(\log n)$-approximate MST can be computed in $O(\log n)$ rounds. The distributed approximation algorithm of Khan and Pandurangan is message-optimal but not time-optimal.

Das Sarma et al. [7] settled the time complexity of distributed approximate MST by showing that this problem, as well as approximating shortest paths and about twenty other problems, satisfies a time lower bound of $\tilde{\Omega}(D+\sqrt{n})$. This applies to deterministic as well as randomized algorithms, and to both exact and approximate versions. In other words, any distributed algorithm for computing a $H$-approximation to MST, for any $H \geq 1$, takes $\tilde{\Omega}(D+\sqrt{n})$ time in the worst case.

Message Complexity. Kutten et al. [31] fully settled the message complexity of leader election in general graphs, even for randomized algorithms and under very general settings. Specifically, they showed that any randomized algorithm (including Monte Carlo algorithms with suitably large constant success probability) requires $\Omega(m)$ messages; this lower bound holds for any $n$ and $m$, i.e., given any $n$ and $m$, there exists a graph with $\Theta(n)$ nodes and $\Theta(m)$ edges for which the lower bound applies. Since a distributed MST algorithm can also be used to elect a leader (where the root of the tree is the leader, which can be chosen using $O(n)$ messages once a tree is constructed), the above lower bound applies to distributed MST construction as well, for all $m \geq c n$, where $c$ is a sufficiently large constant.

The above bound holds even for non-comparison algorithms, that is algorithms that may also manipulate the actual value of node's identities, not just compare identities with each other, and even if nodes have initial knowledge of $n, m$, and $D$. It also holds for synchronous networks, and even if all the nodes wake up simultaneously. Finally, it holds not only for the CONGEST model [45], where sending a message of $O(\log n)$ bits takes one unit of time, but also for the LOCAL model [45], where the number of bits carried in a single message can be arbitrary. On the other hand, it can be shown that an MST can be constructed using $O(m)$ messages (but time can be arbitrarily large) in any synchronous network $[31,41]$.

The $K T_{1}$ Variant. It is important to point out that this article and all the prior results discussed above (including the prior MST results [2, 5, 9, 13-15, 32]) assume the so-called clean network model, a.k.a. $K T_{0}$ [45] (cf. Section 1.1), where nodes do not have initial knowledge of the identity of their neighbors. However, one can assume a model where nodes do have such a knowledge. This model is called the $K T_{1}$ model. Although the distinction between $K T_{0}$ and $K T_{1}$ has clearly no bearing on the asymptotic bounds for the time complexity, it is significant when considering message complexity. Awerbuch et al. [3] show that $\Omega(m)$ is a message lower bound for MST in the $K T_{1}$ model, if one allows only (possibly randomized Monte Carlo) comparison-based algorithms, i.e., 
algorithms that can operate on IDs only by comparing them. (We note that all prior MST algorithms mentioned earlier are comparison-based, including ours.) Hence, the result of [3] implies that our MST algorithm (which is comparison-based and randomized) is time- and message-optimal in the $K T_{1}$ model if one considers comparison-based algorithms only.

Awerbuch et al. [3] also show that the $\Omega(m)$ message lower bound applies even to noncomparison based (in particular, algorithms that can perform arbitrary local computations) deterministic algorithms in the CONGEST model that terminate in a time bound that depends only on the graph topology (e.g., a function of $n$ ). On the other hand, for randomized non-comparison-based algorithms, it turns out that the message lower bound of $\Omega(m)$ does not apply in the $K T_{1}$ model. Recently, King et al. [26] showed a surprising and elegant result: in the $K T_{1}$ model one can give a randomized Monte Carlo algorithm to construct an MST in $\tilde{O}(n)$ messages $(\Omega(n)$ is a message lower bound) and in $\tilde{O}(n)$ time. This algorithm is randomized and not comparison-based. While this algorithm shows that one can achieve $o(m)$ message complexity (when $m=\omega(n$ polylog $n)$ ), it is not time-optimal-it can take significantly more than $\tilde{\Theta}(D+\sqrt{n})$ rounds. In subsequent work, Mashreghi and King [36] presented another randomized, not comparison-based MST algorithm with round complexity $\tilde{O}(\operatorname{Diam}(\mathrm{MST}))$ and with message complexity $\tilde{O}(n)$. Very recently, new algorithms with improved round complexity, but with worse bounds on the message complexity, have been designed $[17,19]$. It is an open question whether one can design a randomized (noncomparison-based) algorithm that takes $\tilde{O}(D+\sqrt{n})$ time and $\tilde{O}(n)$ messages in the $K T_{1}$ model.

\subsection{Subsequent Work}

The preliminary version of this article [42] raised the open problem of whether there exists a deterministic time- and message-optimal MST algorithm. We notice that our algorithm is randomized, due to the use of the randomized cover construction of [9], even though the rest of the algorithm is deterministic. Elkin [11], building on our work, answered this question affirmatively by devising a deterministic MST algorithm that achieves essentially the same bounds as in this article, i.e., uses $\tilde{O}(m)$ messages and runs in $\tilde{O}(D+\sqrt{n})$ time. $^{6}$

The main novelty in Elkin's algorithm is to grow fragments up to diameter $O(D)$, as opposed to $O(\sqrt{n})$, in the first phase of the algorithm. This results in an $(O(n / D), O(D))$-MST forest as the base forest, as opposed to an $(O(\sqrt{n}), O(\sqrt{n}))$-MST forest. Our algorithm is then executed on top of this base forest. This simple change brings benefits to the case $D \geq \sqrt{n}$, for which now the complexities of finding minimum-weight outgoing edges and of their subsequent upcast to the root of the auxiliary BFS tree of the network are within the desired time and message bounds. Hence, Phase 2 of Part 2 of our algorithm is bypassed, and since this phase contains the sole randomized portion of the algorithm (that is, the randomized cover construction of [9]), the final result is a fully deterministic algorithm.

Another round- and message-optimal algorithm for MST appeared very recently in [20], as an application of a new distributed algorithm for computing simple functions over each part of a given partition of the network.

\section{HIGH-LEVEL OVERVIEW OF THE ALGORITHM}

The time- and message-optimal distributed MST algorithm of this article builds on prior distributed MST algorithms that were either message-optimal or time-optimal, but not both. We provide a high-level overview of our algorithm and some intuition behind it; we also compare and contrast it with previous MST algorithms. The full description of the algorithm and its analysis are given in Section 3. The algorithm can be divided into two parts as explained next.

\footnotetext{
${ }^{6}$ Additionally, Elkin's bounds improve those of our article by small polylogarithmic factors.
} 


\subsection{First Part: Controlled-GHS}

We first run the so-called Controlled-GHS algorithm, which was first used in the sublinear-time distributed MST algorithm of Garay et al. [15], as well as in the time-optimal algorithm of Kutten and Peleg [32]. Controlled-GHS is the (synchronous version of the) classical Gallager-HumbletSpira (GHS) algorithm [14, 45], with some modifications. We recall that the synchronous GHS algorithm, which is essentially a distributed implementation of Boruvka's algorithm-see, e.g., [45], consists of $O(\log n)$ phases. In the initial phase each node is an MST fragment, by which we mean a connected subgraph of the MST. In each subsequent phase, every MST fragment finds a lightest (i.e., minimum-weight) outgoing edge (LOE)-these edges are guaranteed to be in the MST by the cut property [48]. The MST fragments are merged via the LOEs to form larger MST fragments. The number of phases is $O(\log n)$, since the number of MST fragments gets at least halved in each phase. The message complexity is $O(m+n \log n)$, which is essentially optimal, and the time complexity is $O(n \log n)$. The time complexity is not optimal because much of the communication during a phase uses only the MST fragment edges. Since the diameter of an MST fragment can be as large as $\Omega(n)$ (and this can be significantly larger than the graph diameter $D$ ), the time complexity of the GHS algorithm is not optimal.

The Controlled-GHS algorithm alleviates this situation by controlling the growth of the diameter of the MST fragments during merging. At the end of Controlled-GHS, at most $\sqrt{n}$ fragments remain, each of which has diameter $O(\sqrt{n})$. These are called base fragments. Controlled-GHS can be implemented using $\tilde{O}(m)$ messages in $\tilde{O}(\sqrt{n})$ rounds. (Note that Controlled-GHS as implemented in the time-optimal algorithm of [32] is not message-optimal-the messages exchanged can be $\tilde{O}\left(m+n^{1.5}\right)$; however, a modified version can be implemented using $\tilde{O}(m)$ messages, as explained in Section 3.1.)

\subsection{Second Part: Merging the $\sqrt{n}$ Remaining Fragments}

The second part of our algorithm, after the Controlled-GHS part, is different from the existing time-optimal MST algorithms. The existing time-optimal MST algorithms [9, 32], as well as the algorithm of [15], are not message-optimal since they use the Pipeline procedure of [15, 44]. The Pipeline procedure builds an auxiliary breadth-first search (BFS) tree of the network, collects all the inter-fragment edges (i.e., the edges between the $\sqrt{n}$ MST fragments) at the root of the BFS tree, and then finds the MST locally. The Pipeline algorithm uses the cycle property of the MST [48] to eliminate those inter-fragment edges that cannot belong to the MST en route of their journey to the root. While the Pipeline procedure, due to the pipelining of the edges to the root, takes $O(\sqrt{n})$ time (since there are at most so many MST edges left to be discovered after the end of the first part), it is not message-optimal: it exchanges $O\left(m+n^{1.5}\right)$ messages, since each node in the BFS tree can send up to $O(\sqrt{n})$ edges leading to $O\left(n^{1.5}\right)$ messages overall (the BFS tree construction takes $O(m)$ messages).

Our algorithm uses a different strategy to achieve optimality in both time and messages. The main novelty of our algorithm (Algorithm 1) is how the (at most) $\sqrt{n}$ base fragments which remain at the end of the Controlled-GHS procedure are merged into one resulting fragment (the MST). Unlike previous time-optimal algorithms [9, 15, 32], we do not use the Pipeline procedure of [15, 44], since it is not message-optimal. Instead, we continue to merge fragments, Borůvka-style. Our algorithm uses two main ideas to implement the Borůvka-style merging efficiently. (Merging is achieved by renaming the IDs of the merged fragments to a common ID, i.e., all nodes in the combined fragment will have this common ID.) The first idea is a procedure to efficiently merge when $D$ is small (i.e., $D=O(\sqrt{n})$ ) or when the number of fragments remaining is small (i.e., $O(n / D)$ ). The second idea is to use sparse neighborhood covers and efficient communication between fragments 
to merge fragments when $D$ is large and the number of fragments is large. Accordingly, the second part of our algorithm can be divided into three phases, which are described next.

2.2.1 Phase 1: When $D$ is $O(\sqrt{n})$. Phase 1 can be treated as a special case of Phase 3 (to be described later). However, we describe Phase 1 separately as it helps in the understanding of the other phases as well.

We construct a BFS tree on the entire network, and perform the merging process as follows: Each base fragment finds its LOE by convergecasting within the fragment to the fragment leader. This takes $O(\sqrt{n})$ time and $O(n)$ messages overall. The $O(\sqrt{n})$ LOE edges are sent by the leaders of the respective base fragments to the root by upcasting (see, e.g., [45]). This takes $O(D+\sqrt{n})$ time and $O(D \sqrt{n})$ messages, as each of the $\sqrt{n}$ edges has to traverse up to $D$ edges on the way to the root. The root merges the fragments and sends the renamed fragment IDs to the respective leaders of the base fragments by downcast (which has the same time and message complexity as upcast [45]). The leaders of the base fragments broadcast the new ID to all other nodes in their respective fragments. This takes $O(\sqrt{n})$ messages per fragment and hence $O(n)$ messages overall. Thus, one iteration of the merging can be done in $O(D+\sqrt{n})$ time and using $O(D \sqrt{n})$ messages. Since each iteration reduces the number of fragments by at least half, the number of iterations is $O(\log n)$. At the end of this iteration, several base fragments may share the same label. In subsequent iterations, each base fragment finds its LOE (i.e., the LOE between itself and the other base fragments which do not have the same label) by convergecasting within its own fragment and (the leader of the base fragment) sends the LOE to the root; thus, $O(\sqrt{n})$ edges are sent to the root (one per base fragment), though there is a lesser number of combined fragments (with distinct labels). The root finds the overall LOE of the combined fragments and does the merging. This is still fine, since the time and message complexity per merging iteration is $O(D+\sqrt{n})$ and $O(D \sqrt{n})=O(n)$, respectively, as required.

2.2.2 Phase 2: When $D$ and the Number of Fragments are Large. When $D$ is large (say $n^{1 / 2+\varepsilon}$, for some $0<\varepsilon \leq 1 / 2$ ) and the number of fragments is large (say, $\Theta(\sqrt{n})$ ) the previous approach of merging via the root of the global BFS tree does not work directly, since the message complexity would be $O(D \sqrt{n})$. The second idea addresses this issue: we merge in a manner that respects locality. That is, we merge fragments that are close by using a local leader, such that the LOE edges do not have to travel too far. The high-level idea is to use a hierarchy of sparse neighborhood covers to accomplish the merging. ${ }^{7}$ A sparse neighborhood cover is a decomposition of a graph into a set of overlapping clusters that satisfy suitable properties (see Definition 3.4 in Section 3.4). The main intuitions behind using a cover are the following: (1) the clusters of the cover have relatively small diameter (compared to the strong diameter of the fragment, ${ }^{8}$ and is always bounded by $D$ ), and this allows efficient communication for fragments contained within a cluster (i.e., the weak diameter of the fragment is bounded by the cluster diameter); (2) the clusters of a cover overlap only a little, i.e., each vertex belongs only to a few clusters; this allows essentially congestion-free (overhead is at most $\operatorname{polylog}(n)$ per vertex) communication and hence operations can be done efficiently in parallel across all the clusters of a cover. This phase continues until the number of fragments reduces to $O(n / D)$, when we switch to Phase 3. We next give more details on the merging process in Phase 2.

\footnotetext{
${ }^{7}$ We use an efficient randomized cover construction algorithm due to Elkin [9]; this is the only randomization used in our algorithm. Neighborhood covers were used by Elkin [9] to improve the running time of the Pipeline procedure of his distributed MST algorithm; on the other hand, here we use them to replace the Pipeline part entirely in order to achieve message optimality as well.

${ }^{8}$ Recall that the strong diameter $\operatorname{diam}_{F}(F)$ of fragment $F$ refers to the longest shortest path (ignoring weights) between any two vertices in $F$ that only passes through vertices in $V(F)$, whereas the weak diameter $\operatorname{diam}_{G}(F)$ allows the use of vertices that are in $V(G) \backslash V(F)$.
} 
Communication-Efficient Paths. An important technical aspect of the merging process is the construction of efficient communication paths between nearby fragments, which are maintained and updated by the algorithm in each iteration. The algorithm requires fragments to be "communication-efficient", in the sense that there is an additional set of short paths between the leader of a fragment $F$ and the fragment members. Such a path might use "shortcuts" through vertices in $V(G) \backslash V(F)$ to reduce the distance. The following definition formalizes this idea:

Definition 2.1 (Communication-Efficient Fragment and Path). Let $F$ be a fragment of $G$, and let $f \in F$ be a vertex designated as the fragment leader of $F$. We say that fragment $F$ is communicationefficient if, for each vertex $v \in F$, it is associated to a path between $v$ and $f$ (possibly including vertices in $V(G) \backslash V(F))$ of length $O\left(\operatorname{diam}_{G}(F)+\sqrt{n}\right)$, where $\operatorname{diam}_{G}(F)$ is the weak diameter of $F$. Such a path is called communication-efficient path for $F$.

Section 3.2 defines the routing data structures that are used to maintain communication-efficient paths. Later, in Section 3.4, we describe the construction of the paths (and routing data structures) inductively. We show that, in each iteration, all fragments find their respective LOEs in time $\tilde{O}(\sqrt{n}+D)$ and using a total of $\tilde{O}(m)$ messages. While we cannot merge all fragments (along their LOEs), as this will create long chains, we use a procedure called ComputeMaximalMatching (Section 3.5) to merge fragments in a controlled manner. ComputeMaximalMatching finds a maximal matching in the fragment graph $\mathcal{F}_{i}$ induced by the LOE edges. The crucial part is using communication-efficient paths to communicate efficiently (both time- and message-wise) between the fragment leader and the nodes in the fragment (while finding LOEs) as well as between fragment leaders of adjacent fragments (while merging as well as implementing ComputeMaximalMatching). The procedure FindLightest (see Section 3.3) describes the LOE finding process assuming communication-efficient fragments. The maintenance of such efficient fragments is shown recursively: the base fragments are efficient and after merging the resulting fragments are also efficient.

We use a hierarchy of sparse neighborhood covers to construct communication-efficient fragments (see Section 3.4). Each cover in the hierarchy consists of a collection of clusters of a certain radius: the lowest cover in the hierarchy has clusters of radius $O(\sqrt{n})$ (large enough to contain at least one base fragment, which has radius $O(\sqrt{n})$ ); subsequent covers in the hierarchy have clusters of geometrically increasing radii, and the last cover in the hierarchy is simply the BFS tree of the entire graph. Initially, it is easy to construct communication-efficient paths in base fragments, since they have strong diameter $O(\sqrt{n})$ (cf. Section 3.2, Lemma 3.2). In subsequent iterations, when merging two adjacent fragments, the algorithm finds a cluster that is (just) large enough to contain both the fragments. Figure 1 in Section 3.4 gives an example of this process. The neighborhood property of the cluster allows the algorithm to construct communication-efficient paths between merged fragments (that might take shortcuts outside the fragments, and hence have small weak diameter) assuming that the fragments before merging are efficient. Note that it is important to make sure that the number of fragments in a cluster is not too large in relation to the radius of the cluster-otherwise, the message complexity would be high (as in the Pipeline scenario). Hence, a key invariant maintained through all the iterations is that the cluster depth times the number of fragments that are contained in the cluster of such depth is always bounded by $\tilde{O}(n)$, and this helps in keeping the message complexity low. This invariant is maintained by making sure that the number of fragments per cluster goes down enough to compensate for the increase in cluster radius (Lemma 3.7 in Section 3.4). At the end of Phase 3, the invariant guarantees that when the cluster radius is $D$, the number of fragments is $O(n / D)$. 
2.2.3 Phase 3: When the Cluster Radius Is $D$. When the cluster radius becomes $D$ (i.e., the cover is just the BFS tree), we switch to Phase 3. The number of remaining fragments will be $O(n / D)$ (which is guaranteed at the end of Phase 2). Phase 3 uses a merging procedure very similar to that of Phase 1. In Phase 1, in every merging iteration, each base fragment finds its respective LOEs, i.e., LOEs between itself and the rest of the fragments, by convergecasting to their respective leaders; the leaders send at most $O(\sqrt{n})$ edges to the root by upcast. The root merges the fragments and sends out the merged information to the base fragment leaders by downcast. In Phase 3 , we treat the $O(n / D)$ remaining fragments as the "base fragments" and repeat the above process. An important difference to Phase 1 is that the merging leaves the leaders of these base fragments intact: in the future iterations of Phase 3, each of these base fragments again tries to find an LOE using the procedure FindLightest, whereby only edges that have endpoints in fragments with distinct labels are considered as candidate for the LOE.

Note that the fragment leaders communicate with their respective nodes as well as the BFS root via the hierarchy of communication-efficient routing paths constructed in Phase 2; these incur only a polylogarithmic overhead. This takes $\tilde{O}(D+n / D)$ time (per merging iteration) since $O(n / D)$ LOE edges are sent to the root of the BFS tree via communication-efficient paths (in every merging iteration) and a message complexity of $\tilde{O}(D \cdot n / D)=\tilde{O}(n)$ (per merging iteration) since, in each iteration, each of the $O(n / D)$ edges takes $\tilde{O}(D)$ messages to reach the root. Since there are $O(\log n)$ iterations overall, we obtain the desired bounds.

\section{DESCRIPTION AND ANALYSIS OF THE ALGORITHM}

The algorithm operates on the MST forest, which is a partition of the vertices of a graph into a collection of trees $\left\{T_{1}, \ldots, T_{\ell}\right\}$ where every tree is a subgraph of the (final) MST. A fragment $F_{i}$ is the subgraph induced by $V\left(T_{i}\right)$ in $G$. We say that an MST forest is an $(\alpha, \beta)$-MST forest if it contains at most $\alpha$ fragments, each with a strong diameter of at most $\beta$. Similarly, an MST forest is a weak $(\alpha, \beta)$-MST forest if it contains at most $\alpha$ fragments, each with a weak diameter of at most $\beta$.

We define the fragment graph, a structure that is used throughout the algorithm. The fragment graph $\mathcal{F}_{i}$ consists of vertices $\left\{F_{1}, \ldots, F_{k}\right\}$, where each $F_{j}(1 \leq j \leq k)$ is a fragment at the start of iteration $i \geq 1$ of the algorithm. The edges of $\mathcal{F}_{i}$ are obtained by contracting the vertices of each $F_{j} \in V(\mathcal{F})$ to a single vertex in $G$ and removing all resulting self-loops of $G$. We sometimes call the remaining edges inter-fragment edges. As our algorithm proceeds by finding lightest outgoing edges (LOEs) from each fragment, we operate partly on the LOE graph $\mathcal{M}_{i}$ of iteration $i$, which shares the same vertex set as $\mathcal{F}_{i}$, i.e., $\mathcal{M}_{i} \subseteq \mathcal{F}_{i}$, but where we remove all inter-fragment edges except for one (unique) LOE per fragment.

\subsection{The Controlled-GHS Procedure}

Our algorithm starts out by making an invocation to the Controlled-GHS procedure introduced in [15] and subsequently refined in [32] and in [33] and [34].

Controlled-GHS (Algorithm 2) is a modified variant of the original GHS algorithm, whose purpose is to produce a balanced outcome in terms of number and diameter of the resulting fragments (whereas the original GHS algorithm allows an uncontrolled growth of fragments). This is achieved by computing, in each phase, a maximal matching on the fragment forest, and merging fragments accordingly. Here, we shall resort to the newest variant presented in [33] and [34], since it incurs a lower message complexity than the two preceding versions. Each phase essentially reduces the number of fragments by a factor of two, while not increasing the diameter of any fragment by more than a factor of two. Since the number of phases of Controlled-GHS is capped at $\lceil\log \sqrt{n}\rceil,{ }^{9}$

\footnotetext{
${ }^{9}$ Throughout, log denotes logarithm to the base 2 .
} 


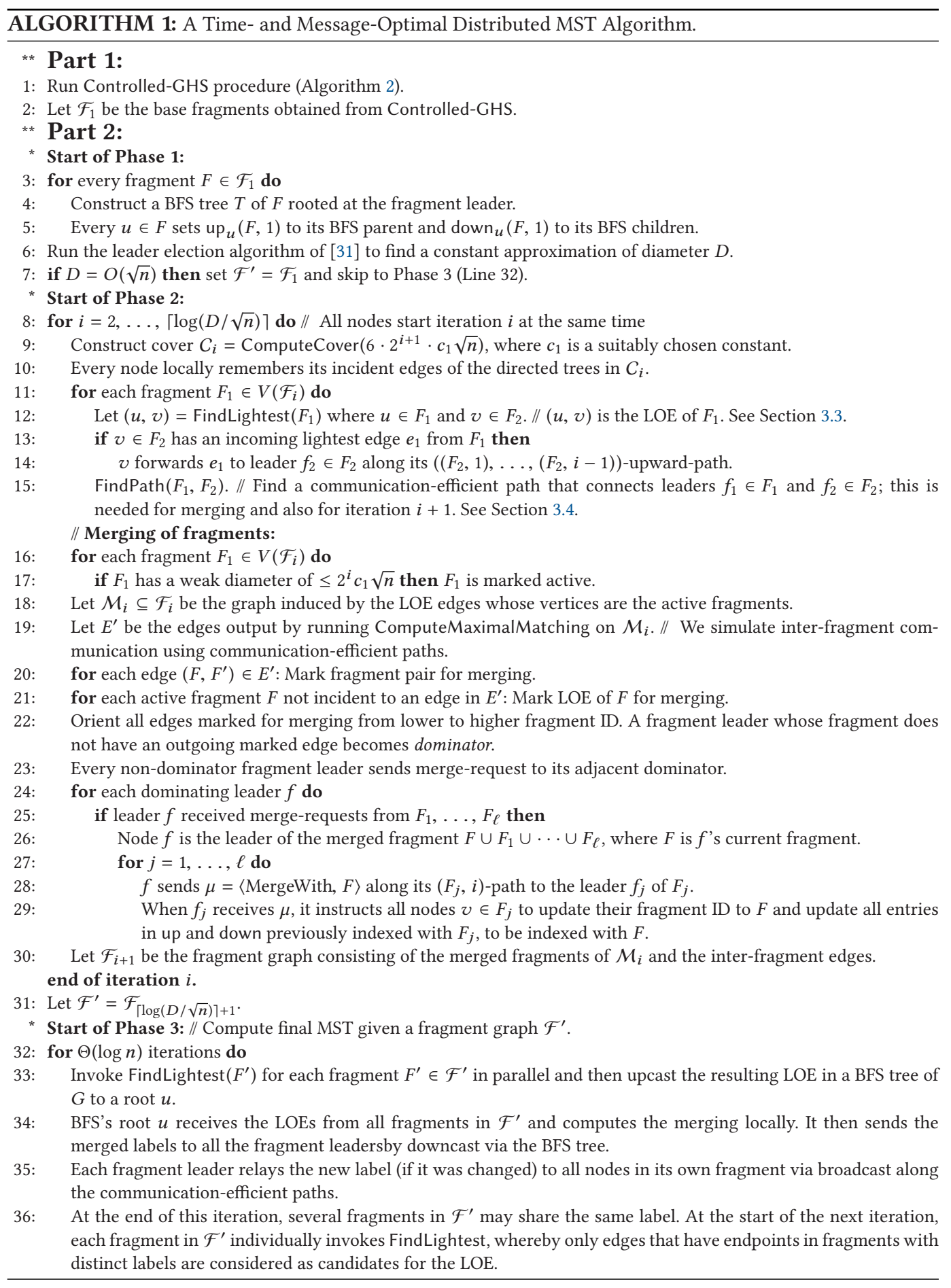




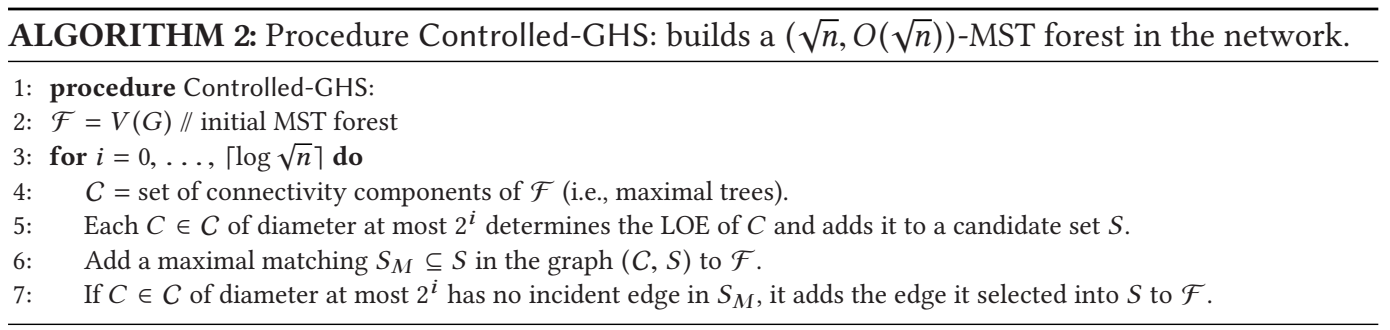

it produces a $(\sqrt{n}, O(\sqrt{n}))$-MST forest. The fragments returned by the Controlled-GHS procedure are called base fragments, and we denote their set by $\mathcal{F}_{1}$.

The following lemma follows from some results in [33], described also in [40].

Lemma 3.1. Algorithm 2 outputs a $(\sqrt{n}, O(\sqrt{n}))$-MST forest in $O\left(\sqrt{n} \log ^{*} n\right)$ rounds and sends $O\left(m \log n+n \log ^{2} n\right)$ messages.

Proof. The correctness of the algorithm is established by Lemma 6.15 and Lemma 6.17 of [33]. By Corollary 6.16 of [33], the $i$ th iteration of the algorithm can be implemented in time $O\left(2^{i} \log ^{*} n\right)$. Hence, the time complexity of Controlled-GHS is

$$
O\left(\sum_{i=0}^{\lceil\log \sqrt{n}\rceil} 2^{i} \log ^{*} n\right)=O\left(\sqrt{n} \log ^{*} n\right)
$$

rounds.

We now analyze the message complexity of the algorithm. Consider any of the $\lceil\log \sqrt{n}\rceil$ iterations of the algorithm. The message complexity for finding the lightest outgoing edge for each fragment (Line 5) is $O(m)$-this follows from the analysis of the GHS algorithms. Then (Line 6) a maximal matching is built using the Cole-Vishkin symmetry-breaking algorithm [6]. As argued in the proof of Corollary 6.16 of [33], in every iteration of this algorithm, only one message per fragment needs to be exchanged. Since the Cole-Vishkin algorithm terminates in $O\left(\log ^{*} n\right)$ iterations, the message complexity for building the maximal matching is $O\left(n \log ^{*} n\right)$. Afterwards, adding selected edges into $S$ to $\mathcal{F}$ (Line 7) can be done with an additional $O(n \log n)$ message complexity. The message complexity of algorithm Controlled-GHS is therefore $O\left(m \log n+n \log ^{2} n\right)$.

\subsection{Routing Data Structures for Communication-Efficient Paths}

For achieving our complexity bounds, our algorithm maintains efficient fragments in each iteration. To this end, nodes locally maintain routing tables. In more detail, every node $u \in G$ has 2 two-dimensional arrays up and down $_{u}$ (called routing arrays), which are indexed by a (fragment ID,level)-pair, where level stands for the iteration number, i.e., the for loop variable $i$ in Algorithm 1. Array up $\mathrm{u}_{u}$ maps to one of the port numbers in $\left\{1, \ldots, d_{u}\right\}$, where $d_{u}$ is the degree of $u$. In contrast, array down $\mathrm{n}_{u}$ maps to a set of port numbers. Intuitively speaking, $\operatorname{up}_{u}(F, i)$ refers to $u$ 's parent on a path $p$ towards the leader of $F$ where $i$ refers to the iteration in which this path was constructed. Similarly, we can think of down $u(F, i)$ as the set of $u$ 's children in all communication efficient paths originating at the leader of $F$ and going through $u$ and we use down $\mathrm{n}_{u}$ to disseminate information from the leader to the fragment members. Oversimplifying, we can envision $u_{u}$ and down $u$ as a way to keep track of the parent-child relations in a tree that is rooted at the fragment leader. (Note that level is an integer in the range $[1,[\log (D / \sqrt{n})\rceil]$ that corresponds to the iteration number of the main loop in which this entry was added; see Lines 8-30 of Algorithm 1.) For a fixed fragment $F$ and some value level $=i$, we will show that the up and down arrays induce directed chains of incident edges. 
Depending on whether we use array up or array down to route along a chain of edges, we call the chain an $(F, i)$-upward-path or an $(F, i)$-downward-path. When we just want to emphasize the existence of a path between a node $v$ and a fragment leader $f$, we simply say that there is a communication-efficient $(F, i)$-path between $v$ and $f$ and we omit " $(F, i)$ " when it is not relevant. We define the nodes specified by $\operatorname{down}_{u}(F, i)$ to be the $(F, i)$-children of $u$ and the node connected to port up $_{u}(F, i)$ to be the $(F, i)$-parent of $u$. So far, we have only presented the definitions of our routing structures. We will explain their construction in more detail in Section 3.4.

We now describe the routing of messages in more detail: Suppose that $u \in F$ generates a message $\mu$ that it wants to send to the leader of $F$. Then, $u$ encapsulates $\mu$ together with $F$ 's ID, the value level $=1$, and an indicator "up" in a message and sends it to its neighbor on port $\operatorname{up}_{u}(F, 1)$; for simplicity, we use $F$ to denote both, the fragment and its ID. When node $v$ receives $\mu$ with values $F$ and level $=1$, it looks up up $v(F, 1)$ and, if $\operatorname{up}_{v}(F, 1)=a$ for some integer $a$, then $v$ forwards the (encapsulated) message along the specified port. ${ }^{10}$ This means that $\mu$ is relayed to the root $w$ of the $(F, 1)$-upward-path. For node $w$, the value of $\operatorname{up}_{w}(F, 1)$ is undefined and so $w$ attempts to lookup $\operatorname{up}_{w}(F, 2)$ and then forwards $\mu$ along the $(F, 2)$-upward-path and so forth. In a similar manner, $\mu$ is forwarded along the path segments $p_{1} \ldots p_{i}(1 \leq j \leq i)$, where $p_{j}$ is the $(F, j)$-upward-path in the $i$ th iteration of the algorithm's main-loop. We will show that the root of the $(F, i)$-upward-path coincides with the fragment leader at the start of the $i$ th iteration.

On the other hand, when the iteration leader $u$ in the $i$ th iteration wants to disseminate a message $\mu$ to the fragment members, it sends $\mu$ to every port in the set $\operatorname{down}_{u}(F, i)$. Similarly to above, this message is relayed to each leaf $v$ of each $(F, i)$-downward-path, for which the entry $\operatorname{down}_{v}(F, i)$ is undefined. When $i>1$, node $v$ then forwards $\mu$ to the ports in $\operatorname{down}_{v}(F, j)$, for each $j<i$ for which $v$ is a root of the respective $(F, j)$-upward-path, and $\mu$ traverses the path segments $q_{i} \ldots q_{1}$ where $q_{\ell}(1 \leq \ell \leq i)$ is the $(F, \ell)$-downward-path. For convenience, we call the concatenation of $q_{i} \ldots q_{1}$ a $((F, i), \ldots,(F, 1))$-downward path (or simply $((F, i), \ldots,(F, 1))$-path), and define a $((F, 1), \ldots,(F, i))$-upward path similarly.

We are now ready to describe the individual components of our algorithm in more detail. To simplify the presentation, we will discuss the details of Algorithm 1 inductively. We assume that every node $u \in F \in \mathcal{F}_{1}$ knows its parent and children in a BFS tree rooted at the fragment leader $f \in F$. (BFS trees for spanning each fragment can easily be constructed: in fact, the Controlled-GHS procedure provides trees of depth $O(\sqrt{n})$ for each base fragment.) Thus, node $u$ initializes its routing arrays by pointing $\operatorname{up}_{u}(F, 1)$ to its BFS parent and by setting $\operatorname{down}_{u}(F, 1)$ to the port values connecting its BFS children.

Lemma 3.2. At the start of the first iteration of the algorithm, for any fragment $F$ and every $u \in F$, there is an $(F, 1)$-path between $F$ 's fragment leader and $u$ with a path length of $O(\sqrt{n})$.

Proof. From the initialization of the routing tables up and down it is immediate that we reach the leader when starting at a node $u \in F$ and moving along the $(F, 1)$-upward-path. Similarly, starting at the leader and moving along the $(F, 1)$-downward-path, allows us to reach any fragment member. The bound on the path length follows from the strong diameter bound of the base fragments, i.e., $O(\sqrt{n})$ (see Lemma 3.1).

\subsection{Finding the Lightest Outgoing Edges (LOEs): Procedure FindLightest}

We now describe Procedure FindLightest $(F)$, which enables the fragment leader $f$ to obtain the lightest outgoing edge, i.e., the lightest edge that has exactly one endpoint in $F$. Consider iteration

\footnotetext{
${ }^{10}$ Node $v$ is free to perform additional computations on the received messages as described by our algorithms, e.g., $v$ might aggregate simultaneously received messages in some form. Here we only focus on the forwarding mechanism.
} 
$i \geq 1$. As a first step, FindLightest $(F)$ requires all fragment nodes to exchange their fragment IDs with their neighbors to ensure that every node $v$ knows its set of incident outgoing edges $E_{v}$. If a node $v$ is a leaf in the BFS tree of its base fragment, i.e., it does not have any $(F, 1)$-children, it starts by sending the lightest edge in $E_{v}$ along the $((F, 1), \ldots,(F, i))$-upward-path. In general, a node $u$ on an $(F, j)$-upward-path $(j \geq 1)$ waits to receive the lightest-edge messages from all its $(F, j)$-children (or its $(F, j-1)$-children if any), and then forwards the lightest outgoing edge that it has seen to its parent in the $((F, j), \ldots,(F, i))$-upward-path.

The following lemma proves some useful properties of FindLightest. Note that we do not yet claim any bound on the message complexity at this point, as this requires us to inductively argue on the structure of the fragments, which relies on properties that we introduce in the subsequent sections. Hence, we postpone the message complexity analysis to Lemma 3.12.

Lemma 3.3 (EFficient LOE Computation). Suppose that at the start of iteration $i+1 \geq 2$ every fragment in $F \in \mathcal{F}_{i}$ is communication-efficient. Then, the fragment leader of $F$ obtains the lightest outgoing edge by executing Procedure FindLightest $(F)$ in $O\left(\operatorname{diam}_{G}(F)+\sqrt{n}\right)$ rounds.

Proof. To accurately bound the congestion, we must consider the simultaneous invocations of FindLightest for each fragment in $\mathcal{F}_{i}$. Since, by assumption, every fragment is communicationefficient, every fragment node $u$ can relay its lightest outgoing edge information to the fragment leader along a path $p$ of length $O\left(\operatorname{diam}_{G}(F)+\sqrt{n}\right)$. Note that $p$ is precisely the $((F, 1), \ldots,(F, i))$ upward path to the leader starting at $u$. To bound the congestion, we observe that the $(F, 1)$ upward subpath of $p$ is confined to nodes in $F_{u}$ where $F_{u}$ is the base fragment that $u$ was part of after executing Controlled-GHS. As all base fragments are disjoint and lightest edge messages are aggregated within the same base fragment, the base fragment leader (who might not be the leader of the current fragment $F$ ) accumulates this information from nodes in $F_{u}$ within $O(\sqrt{n})$ rounds (cf. Lemma 3.2). After having traversed the $(F, 1)$-upward path (i.e., the first segment of $p$ ) of each base fragment, the number of distinct messages carrying lightest edge information is reduced to $O(\sqrt{n})$ in total. Hence, when forwarding any such message along a subsequent segment of $p$, i.e., an $\left(F_{j}\right)$-upward path for $j>1$, the maximum congestion at any node can be $O(\sqrt{n})$. Using a standard upcast (see, e.g., [45]) and the fact that the length of path $p$ is $O\left(\operatorname{diam}_{G}(F)+\sqrt{n}\right)$, it follows that the fragment leader receives all messages in $O\left(\operatorname{diam}_{G}(F)+\sqrt{n}\right)$ rounds, as required.

\subsection{Finding Communication-Efficient Paths: Procedure FindPath}

After executing FindLightest $\left(F_{1}\right)$, the leader $f_{1}$ of $F_{1}$ has obtained the identity of the lightest outgoing edge $e=(u, v)$ where $v$ is in some distinct fragment $F_{2}$. Before invoking our next building block, Procedure FindPath $\left(F_{1}, F_{2}\right)$, we need to ensure that both leaders are aware of $e$ and hence we instruct the node $v$ to forward $e$ along its $\left(\left(F_{2}, 1\right), \ldots,\left(F_{2}, i\right)\right)$-upward-path to its leader $f_{2}$ (see Lines 13-14 of Algorithm 1).

We now describe FindPath $\left(F_{1}, F_{2}\right)$ in detail. The goal is to compute a communication-efficient path between leaders $f_{1}$ and $f_{2}$ that can be used to route messages between nodes in this fragment. In Section 3.5, we will see how to leverage these communication-efficient paths to efficiently merge fragments.

A crucial building block for finding an efficient path are the sparse neighborhood covers that we precompute at the start of each iteration (see Line 9 of Algorithm 1), and the properties of which we recall here. Note that the cover definition concerns the underlying unweighted graph, i.e., all distances are just the hop distances.

Definition 3.4. A sparse $(\kappa, W)$-neighborhood cover of a graph is a collection $C$ of trees, each called a cluster, with the following properties. 
(1) (Depth property) For each tree $\tau \in C$, depth $(\tau)=O(W \cdot \kappa)$.

(2) (Sparsity property) Each vertex $v$ of the graph appears in $\tilde{O}\left(\kappa \cdot n^{1 / \kappa}\right)$ different trees $\tau \in C$.

(3) (Neighborhood property) For each vertex $v$ of the graph there exists a tree $\tau \in C$ that contains the entire $W$-neighborhood of vertex $v$.

Sparse neighborhood covers were introduced in [4], and were found useful in several applications. We will use an efficient distributed (randomized) cover construction due to Elkin [9], which we recall here. ${ }^{11}$

Theorem 3.5 ([9, Theorem A.8]). There exists a distributed randomized Las Vegas algorithm, which here we call ComputeCover, that constructs $a(\kappa, W)$-neighborhood cover in time $O\left(\kappa^{2} \cdot n^{1 / \kappa}\right.$. $\log n \cdot W)$ and using $O\left(m \cdot \kappa \cdot n^{1 / \kappa} \cdot \log n\right)$ messages (both bounds hold with high probability) in the CONGEST model.

In our MST algorithm, we shall invoke Elkin's ComputeCover procedure with $\kappa=\log n$, and write ComputeCover $(W)$, where $W$ is the neighborhood parameter.

We are now ready to describe the communication-efficient paths construction. As we want to keep the overall message complexity low, we start at the smallest cover construction $C_{1}$ and carefully probe for a cluster (tree) in $C_{1}$ that induces a communication-efficient path between $f_{1}$ and $f_{2}$. Recall that every node locally keeps track of its incident cluster edges for each of the precomputed covers but we need to keep in mind that these structures are independent of the up and down arrays. We instruct both leaders $f_{1}$ and $f_{2}$ to send a copy of their probe message to each of their $C_{1}$-parents. The parent nodes forward $u$ 's probe message along their cluster tree to the root of their respective cluster tree. Depending on whether a root receives the probe message in a timely fashion, we consider two cases:

Case 1: If there exists $C_{w} \in C_{1}$ such that $f_{1}, f_{2} \in C_{w}$, then the probe message of both leaders reaches, through some path $p_{0}$ and $p_{1}$, the root $w \in C_{w}$ within $\tilde{O}\left(6 \cdot 2^{2} c_{1} \sqrt{n} \log n+\sqrt{n} \log ^{2} n\right)$ rounds, where the first term is depth $\left(C_{1}\right)$ and the second term is to account for the congestion caused by simultaneous probe messages from the other fragment leaders (cf. Lemma 3.8). Then, $w$ replies by sending a "success" message back to $f_{1}$ and $f_{2}$ by reversing paths $p_{1}$ and $p_{2}$ to inform the leaders that they have found a communication-efficient path.

Note that it is possible for $f_{1}$ to receive multiple "success" reply messages. However, since a cluster root only sends a success message if it receives probe messages from both leaders, $f_{1}$ and $f_{2}$ receive exactly the same set $M$ of success messages. Thus, they both pick the same success message sent by the cluster root node with the largest ID in $M$ (without loss of generality, assume that it is $w$ ) to identify the communication-efficient path and discard the other messages in $M$.

Suppose that $f_{1}$ received the message from $w$ along a path $p_{1}$ in cluster tree $C_{w}$. Then, $f_{1}$ sends a message along $p_{1}$ and instructs every node $v$ in $p_{1}$ to set $\operatorname{up}_{v}\left(F_{2}, i+1\right)$ to the port of its successor (towards the root $w$ ) in $p_{1}$ and points $\operatorname{up}_{v}\left(F_{1}, i+1\right)$ to its predecessor in $p_{1}$. When a node $v$ updates its $\operatorname{up}_{v}\left(F_{2}, i+1\right)$ array to some port $a$, it contacts the adjacent node $v^{\prime}$ connected at this port who in turn updates down $v_{v^{\prime}}\left(F_{2}, i+1\right)$ to point to $v$. Similarly, leader $f_{2}$ and all nodes on the path $p_{2}$ proceeds updating their respective up and down entries with the information provided by $p_{2}$ towards $w$. Then, $f_{1}$ contacts its successor in $p_{1}$ to update its routing information whereas $f_{2}$ sends a similar request to its successor in $p_{2}$. After these requests reach the cluster root $w$, the concatenated path $p_{1} p_{2}$ is a communication-efficient path between leaders $f_{1}$ and $f_{2}$.

\footnotetext{
${ }^{11}$ Although the algorithm as described in [9] is Monte Carlo, it can be easily converted to Las Vegas.
} 


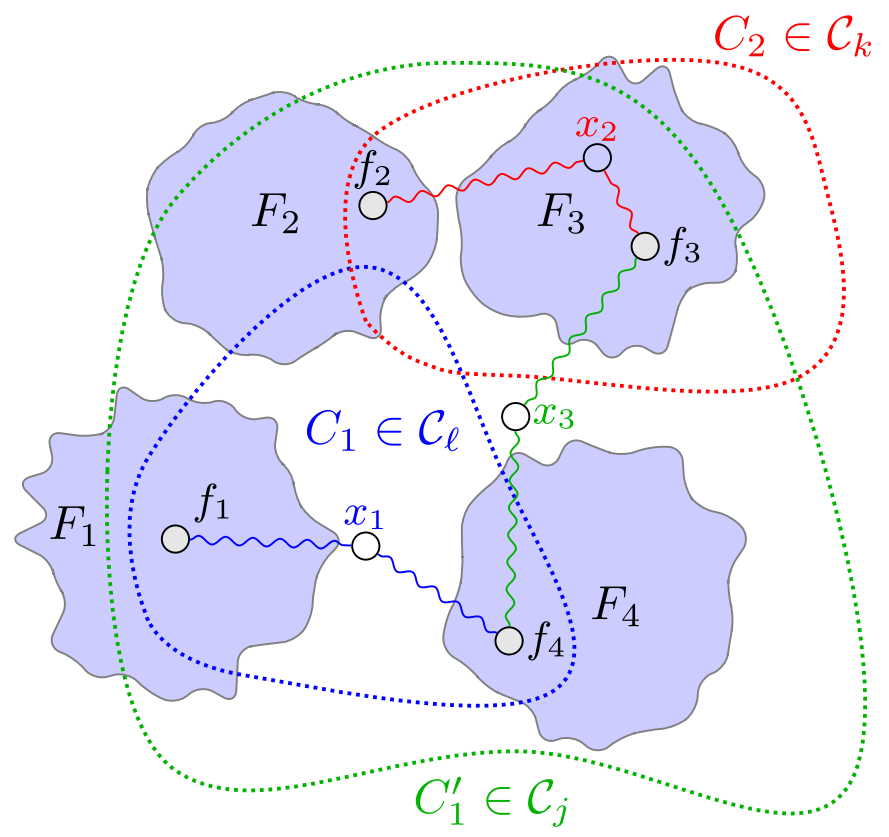

Fig. 1. Fragments $F_{1}, \ldots, F_{4}$. In the first iteration, $F_{1}, F_{4}$ and $F_{2}, F_{3}$ form adjacent fragment pairs that communicate along communication-efficient paths. $F_{1}$ and $F_{4}$ execute FindPath and send probe messages along clusters of covers $C_{1}, \ldots, C_{\ell}$ and finally succeed to find a communication-efficient path in a cluster $C_{1} \in C_{\ell}$, which goes through the cluster leader $x_{1} \in C_{1}$. Similarly $F_{2}$ and $F_{3}$ obtain a communication-efficient path in cluster $C_{2} \in C_{k}$, after sending probe messages in clusters of covers $C_{1}, \ldots, C_{k}$. In the next iteration, the merged fragments $F_{1} \cup F_{4}$ and $F_{2} \cup F_{3}$ are (respectively) adjacent and proceed to construct a communicationefficient path in cluster $C_{1}^{\prime} \in C_{j}$, after probing covers $C_{1}, \ldots, C_{j}$.

Case 2: On the other hand, if there is no appropriate cluster in $C_{1}$ that covers both leader nodes, then at least one of the two probe messages will arrive untimely at every cluster root and the leaders do not receive any success messages. Then, $f_{1}$ and $f_{2}$ rerun the probing process by sending a probe message along their incident $C_{2}$ cluster edges and so forth. Note that all fragment leaders synchronize before executing the probing process. We show in Lemma 3.7 that all fragments have weak diameter at most $6 \cdot 2^{i} c_{1} \sqrt{n}$ in iteration $i$. Notice the radius of $C_{i}$ (see Line 9) ensures that $f_{1}$ and $f_{2}$ will arrive at a value $k \leq i$, where $C_{k}$ is the cover having the smallest depth such that $f_{1}$ and $f_{2}$ are covered by some cluster in $C_{k}$ (but not by any cluster in $C_{k-1}$ ). Thus, we can apply Case 1 for $C_{k}$.

Figure 1 gives an example for the construction of communication-efficient paths.

Lemma 3.6. The number of probe messages that are generated by distinct fragment leaders and that are in transit simultaneously during an iteration of FindPath is $O\left(\sqrt{n} \log ^{2} n\right)$ w.h.p.

Proof. Since, by Lemma 3.1, there are $O(\sqrt{n})$ base fragments, the total number of leaders at any point that are sending probe messages simultaneously is $O(\sqrt{n})$. Note that, when exploring the communication efficient paths of a cover $C_{j}$, a leader needs to send a copy of its probe message to its parent in each of its $O\left(\log ^{2} n\right)$ clusters of $C_{j}$ that it is contained in.

Lemma 3.7. At the start of each iteration $i+1$, the fragment graph $\mathcal{F}_{i}$ induces a weak $\left(\sqrt{n} / 2^{i}, 6\right.$. $\left.\left.2^{i} c_{1} \sqrt{n}\right)\right)-M S T$ forest in $G$. 
Proof. We adapt the proof of Lemmas 6.15 and 6.17 of [33] to show that the fragment graph is a weak $\left(\sqrt{n} / 2^{i}, 6 \cdot 2^{i} c_{1} \sqrt{n}\right)$-MST forest. For the case $i=1$, the claim follows directly from Lemma 3.1. We now focus on the inductive step $i>1$.

Suppose that $\mathcal{F}_{i}$ is a weak $\left(\sqrt{n} / 2^{i}, 6 \cdot 2^{i} c_{1} \sqrt{n}\right)$-MST forest. We first argue that every new fragment in $\mathcal{F}_{i+1}$ must have a weak diameter of at most $6 \cdot 2^{i+1} c_{1} \sqrt{n}$.

Consider the subgraph $M$ of $\mathcal{F}_{i}$ induced by the edges marked for merging. By Lines 20-21 of Algorithm 1, each component of $M$ can contain at most one marked edge that was in the output of ComputeMaximalMatching. Thus, analogously to Lemma 6.15 in [33], it follows that each component in $M$ contains at most one fragment of weak diameter $>2^{i} c_{1} \sqrt{n}$, since only fragments of weak diameter at most $2^{i} c_{1} \sqrt{n}$ become active and participate in the matching. Note that the maximality of the matching implies that each component of $M$ has diameter (in the fragment subgraph $M)$ at most 3. Moreover, all except at most 1 fragment of such a component must have a weak diameter of at most $2^{i} c_{1} \sqrt{n}$ since a fragment of a larger weak diameter does not select any edges for merging in this iteration. It follows by the inductive hypothesis that the merged component has a weak diameter of at most $6 \cdot 2^{i} c_{1} \sqrt{n}+3 \cdot 2^{i} c_{1} \sqrt{n} \leq 6 \cdot 2^{i+1} c_{1} \sqrt{n}$.

We now argue that each fragment contains at least $2^{i} c_{2} \sqrt{n}$ nodes at the start of iteration $i>1$, assuming that it is true for all $j=1, \ldots, i-1$. To this end, consider the merging of fragments in iteration $i-1$. If a fragment $F \in \mathcal{F}_{i}$ contains less than $2^{i} c_{2} \sqrt{n}$ nodes, it must have a weak diameter of at most $2^{i} c_{2} \sqrt{n}$ and hence marks itself as active in Line 17. By the description of the merging process, $F$ is guaranteed to merge with at least one other fragment $F^{\prime}$. By the inductive hypothesis, both $F$ and $F^{\prime}$ consist of at least $2^{i-1} c_{2} \sqrt{n}$ nodes and hence the merged fragment must have at least $2^{i} c_{2} \sqrt{n}$ nodes, as required.

Lemma 3.8. Consider any iteration $i \geq 1$. After the execution of FindPath $\left(F_{1}, F_{2}\right)$, there exists a communication-efficient path between leader $f_{1}$ and leader $f_{2}$ of length at most $O\left(2^{k} \sqrt{n}\right)$, where $k \leq i$ is the smallest integer such that there exists a cluster tree $C \in C_{k}$ such that $f_{1}, f_{2} \in C$. FindPath $\left(F_{1}, F_{2}\right)$ requires $O\left(2^{k} \sqrt{n} \log ^{2} n\right)$ messages and terminates in

$$
\tilde{O}\left(\sqrt{n} \log ^{2} n+\min \left\{2^{k} \sqrt{n}, \operatorname{diam}(G)\right\}\right)
$$

rounds with high probability.

Proof. By the description of FindPath, leaders $f_{1}$ and $f_{2}$ both start sending a probe message along their incident $C_{j}$-edges towards the respective cluster roots, for $j=1, \ldots,\lceil\log \sqrt{n}\rceil$. First, note that $f_{1}$ and $f_{2}$ will not establish an efficient communication path for a cluster $C^{\prime}$ in some $C_{j}$ $(j<k)$, since, by definition, $f_{1}$ and $f_{2}$ are not both in $C^{\prime}$ and hence one of the probe messages will not reach the root of $C^{\prime}$. To see that $k \leq i$, note that Lemma 3.7 tells us that in iteration $i$ every fragment has weak diameter at most $O\left(2^{i} \sqrt{n}\right)$, whereas $C_{i}$ has a cluster radius of $\Theta\left(2^{i+1} \sqrt{n} \log n\right)$.

We now argue the message complexity bound. Apart from the probe messages sent to discover the communication-efficient path in a cluster of $\operatorname{cover} C_{k}$, we also need to account for the probe messages sent along cluster edges of covers $C_{1}, \ldots, C_{k-1}$, thus generating at most

$$
\sum_{j=1}^{k} O\left(\operatorname{depth}\left(C_{j}\right) \log ^{2} n\right)=\sum_{j=1}^{k} O\left(2^{j} \sqrt{n} \log ^{2} n\right) \leq 2^{k+1} O\left(\sqrt{n} \log ^{2} n\right)=O\left(\operatorname{depth}\left(C_{k}\right) \log ^{2} n\right)
$$

messages, as required.

Since $f_{1}$ and $f_{2}$ can communicate efficiently via a path $p$ leading through a cluster of cover $C_{k}$, then the length of $p$ is at most $2 \operatorname{depth}\left(C_{k}\right)$. Applying Lemma 3.6 to take into account the additional congestion caused by simultaneous probe messages, yields a time complexity of $\tilde{O}\left(\operatorname{depth}\left(C_{k}\right)+\right.$ $\left.\sqrt{n} \log ^{2} n\right)$. 
Lemma 3.9. Consider an iteration $i$ and suppose that FindPath is invoked simultaneously for each lightest outgoing edge. Then, the total message complexity of all invocations is $O\left(n \log ^{3} n\right)$ and the time complexity is $\tilde{O}(\operatorname{diam}(G)+\sqrt{n})$ with high probability.

Proof. From Lemma 3.7, we know that every fragment in $\mathcal{F}_{i}$ has weak diameter of $O\left(2^{i} \sqrt{n}\right)$. Thus, every pair of adjacent fragments $F_{1}, F_{2} \in \mathcal{F}_{i}$ is covered by some cluster in cover $C_{i+1}$. In this case, Lemma 3.8 tells us that a single invocation of FindPath requires $O\left(2^{i+1} \sqrt{n} \log ^{2} n\right)$ messages. Lemma 3.7 tells us that there are $O\left(\sqrt{n} / 2^{i}\right)$ fragments in $\mathcal{F}_{i}$ (and thus also $O\left(\sqrt{n} / 2^{i}\right)$ LOEs). Hence, the total number of messages incurred by all pairs of fragments connected by an LOE is

$$
O\left(2^{i+1} \sqrt{n} \log ^{2} n\right) \cdot O\left(\sqrt{n} / 2^{i}\right)=O\left(n \log ^{2} n\right) .
$$

Summing up over all $i$, we obtain the claimed bound on the message complexity.

Finally, we observe that Lemma 3.8 already takes into account the congestion caused by simultaneous invocations, which yields the bound on the time complexity.

To summarize, Procedure FindPath enables leaders of adjacent fragments to communicate with each other by sending messages along the communication-efficient paths given by the routing tables up and down.

\subsection{Merging Fragments}

We will avoid long chains of merged fragments by using procedure ComputeMaximalMatching [33]. ComputeMaximalMatching outputs a maximal matching on a fragment forest, where fragments in $\mathcal{F}_{i}$ are treated as super-vertices of a graph connected by inter-fragment edges. Procedure ComputeMaximalMatching simulates the Cole-Vishkin symmetry-breaking distributed algorithm, which terminates in $O\left(\log ^{*} n\right)$ iterations [33, Theorem 1.7]. We next show how to do the simulation efficiently in the fragment graph.

Procedure FindPath enables communication via communication-efficient paths between adjacent fragment leaders in $\mathcal{M}_{i}$. In turn, this enables the simulation of procedure ComputeMaximalMatching on the network induced by $\mathcal{M}_{i}$, where the leaders in $\mathcal{M}_{i}$ perform the computation required by ComputeMaximalMatching. The following lemma follows directly from Lemma 3.9:

Lemma 3.10. Suppose that every fragment in $\mathcal{F}_{i}$ is communication-efficient and let $\mathcal{M}_{i} \subset \mathcal{F}_{i}$ be the lightest outgoing edge graph obtained by running FindPath. Then, ComputeMaximalMatching can be simulated on the network defined by $\mathcal{M}_{i}$, requiring $\tilde{O}(\operatorname{diam}(G)+\sqrt{n})$ rounds and $\tilde{O}(n)$ messages.

Every non-dominator fragment $F_{1}^{\prime}$ sends a $\langle$ MergeReq $\rangle$ message to the leader $f_{1}^{\prime}$ of an arbitrarily chosen adjacent dominator fragment $F$. The dominator fragment processes all merge-requests in parallel and replies by sending a $\langle$ MergeWith, $F\rangle$ message to the leader $f^{\prime}$ of each fragment $F^{\prime}$ from which it received $\langle$ MergeReq $\rangle$; in turn, $f^{\prime}$ forwards this request along the $\left(\left(F^{\prime}, i\right), \ldots,\left(F^{\prime}, 1\right)\right)$ downward path to every node in $F^{\prime}$. Upon receiving a $\langle$ MergeWith, $F\rangle$ message, node $u^{\prime} \in F^{\prime}$ updates its fragment ID to $F$, and also updates its routing table by setting up $u_{u^{\prime}}(F, \ell)=\operatorname{up}_{u^{\prime}}\left(F^{\prime}, \ell\right)$ and down $_{u^{\prime}}(F, \ell)=$ down $_{u^{\prime}}\left(F^{\prime}, \ell\right)$, for every value of $\ell$. Note that the leader of the dominator fragment becomes the new leader of the merged fragment.

Lemma 3.11. Consider iteration $i$. If, for each $j \leq i$, every fragment in $\mathcal{F}_{j}$ is communication-efficient, then the following hold:

(1) With high probability, the message complexity for merging fragments in iteration $i$ is $\tilde{O}(m)$ and the process completes within $\tilde{O}(\operatorname{diam}(G)+\sqrt{n})$ rounds.

(2) Every fragment in $\mathcal{F}_{i+1}$ is communication-efficient. 
Proof. To show (1), we argue recursively starting at iteration $i$, as follows: note that forwarding the $\langle$ MergeWith $\rangle$ and $\langle$ MergeReq $\rangle$ messages requires communicating between neighboring fragments and thus by Lemma 3.10 we require $O(\operatorname{diam}(G)+\sqrt{n})$ rounds and $O\left(n \log ^{2} n\right)$ messages. Consider an adjacent pair of fragments $F_{0}$ and $F_{1}$ and suppose that $F_{0}$ merges with the dominator fragment $F_{1}$. Since we eventually need to broadcast the new fragment ID to every node $u \in F_{0}$, we need to ensure that the routing tables $\operatorname{up}_{u}\left(F_{1}, \cdot\right)$ and down ${ }_{u}\left(F_{1}, \cdot\right)$ are updated correctly to route messages towards the new leader $f_{1} \in F_{1}$ (and vice-versa from $f_{1}$ to all nodes in $F_{1}$ ), when we compute the lightest outgoing edge of the merged fragment $F_{0} \cup F_{1}$ in subsequent iterations. If $i>1$, then $F_{0}$ might be composed of merged fragments $F_{0}^{\prime} \cup \cdots \cup F_{\ell}^{\prime}$ that merged in previous iterations; without loss of generality, suppose that this iteration is $i-1$. By assumption, $\mathcal{F}_{i-1}$ consisted of efficient fragments. As nodes do not remove routing information from up and down, the leader $f_{0}$ can use the communication-efficient paths obtained by invoking FindPath in iteration $i-1$ to forward the new fragment ID to the leaders of the $F_{0}^{\prime}, \ldots, F_{\ell}^{\prime}$, which we call the $(i-1)$-iteration fragments. Applying Lemma 3.10 to $\mathcal{M}_{i-1}$ reveals that we can use the paths obtained by invoking FindPath in iteration $i-1$ to relay the new fragment ID to $(i-1)$-iteration fragments while incurring only $O(\operatorname{diam}(G)+\sqrt{n})$ rounds and $O\left(n \log ^{2} n\right)$ messages in total. Recursively applying this argument until iteration 1 , allows us to reason that $O((\operatorname{diam}(G)+\sqrt{n}) \log n)$ rounds and $O\left(n \log ^{3} n\right)$ messages are sufficient to relay all new fragment IDs to the base fragment leaders. At this point, every base fragment leader uses the BFS tree of the base fragments to broadcast this information to the base fragment nodes, requiring $O(\sqrt{n})$ rounds and $O(m)$ messages.

To show (2), we observe that $\mathcal{F}_{i}$ consists of communication-efficient fragments, and hence every fragment node $u \in F_{j}$ of a newly merged fragment $F=F_{1} \cup \cdots \cup F_{\ell}(\ell \geq j)$ can already communicate efficiently with the leader $f_{j}$ in its subfragment $F_{j}$, which has now become part of $F$. Moreover, the paths obtained by FindPath ensure that $f_{j}$ can communicate efficiently with leader $f \in F$ and hence it follows transitively that $u$ has a communication-efficient path to $f$, as required.

The analysis of the message complexity of merging fragments allows us to obtain a bound on the number of messages required for computing a lightest outgoing edge in each fragment.

LEMMA 3.12. The total message complexity of all parallel invocations of FindLightest is Õ $(m)$ w.h.p.

Proof. In the first step of FindLightest, each node exchanges messages with its neighbors requiring $\Theta(m)$ messages. Let $F=F_{1} \cup \cdots \cup F_{\ell}$, where $F_{1}, \ldots, F_{\ell}$ are base fragments, and consider some vertex $u \in F_{1}$. As previously argued, $u$ relays its LOE information along the $((F, 1), \ldots,(F, i))$-upward-path to the fragment leader and the segment formed by the $(F, 1)$ upward path ends at the base fragment leader of $F_{1}$, which are exactly the BFS trees yielded by Controlled-GHS. A crucial observation is that $u$ only sends its LOE information to its parent in the path, after receiving the LOE messages from all its children (see Section 3.3). This ensures that each node sends exactly one message and hence we obtain a bound of $\sum_{j=1}^{\ell} O\left(\left|V\left(F_{j}\right)\right|\right)=O(|V(F)|)$ on the number of messages sent in the $(F, 1)$-upward-path of the nodes in $F$. This is subsumed in the message complexity of exchanging messages with neighbors in the first step, which is $O(m)$.

At this point, each base fragment leader $f_{j}$ of $F_{j}(j=1, \ldots, \ell)$ holds exactly one (aggregated) lightest outgoing edge information message $\mu_{j}$, which needs to be relayed to the fragment leader $f$ of $F$ along the respective $((F, 2), \ldots,(F, i))$-upward-path of $O\left(\operatorname{diam}_{G}(F)\right)$ hops (see Definition 2.1).

By reversing the argument used for proving part (2) of Lemma 3.11, we can inductively apply Lemma 3.10 to obtain a bound of $O\left(n \log ^{3} n\right)$ messages per iteration, and thus the total message complexity is $O\left(m+n \log ^{3} n\right)=\tilde{O}(m)$.

We now analyze the correctness and the complexities of Phase 3 of the algorithm. 
Lemma 3.13. Phase 3 of the algorithm requires $\tilde{O}(m)$ messages and $\tilde{O}(D+\sqrt{n})$ time and ensures that all fragments have the same label (i.e., are merged).

Proof. Note that our algorithm either executes Phase 3 directly after Phase 1 (thus skipping Phase 2) or after executing Phase 2. First, we argue (for both cases) that all fragments have the same fragment ID after the $\Theta(\log n)$ iterations in Phase 3. To see that the number of fragment labels is at least halved in each iteration, note that, when executing FindLightest, all nodes exchange their fragment IDs with their neighbors (requiring $O(m)$ messages) and then only choose candidate LOE edges that have their endpoint in fragments with distinct IDs. This ensures that every fragment pairs up with another fragment and hence one of the two distinct IDs will be removed; note that long "chains" of fragments connected by LOE edges are possible and result in an even faster reduction of distinct labels-all fragments in the chain adapt the root fragment ID (cf. Phase 3 in the pseudo code). Thus, after the last iteration of Phase 3, all fragments carry the same fragment ID and no more LOE edges are required as all fragments are considered to be merged.

Now we consider the message and time complexity of Phase 3. According to Lemma 3.3, the time complexity of finding the LOEs is $O(D+\sqrt{n})$, and according to Lemma 3.12, $\tilde{O}(m)$ messages are required to find the LOEs. This is true independently of whether we called Phase 3 directly after Phase 1 or after Phase 2.

Now, consider the case where we execute Phase 3 directly after Phase 1 (thus skipping Phase 2), i.e., $D=O(\sqrt{n})$. Here, FindLightest results in each node locally determining the incident LOE and then aggregating the LOE to the base fragment leader. In addition to the base fragment BFS trees, we also construct a global BFS tree $T$, which, has $O(\sqrt{n})$ diameter by assumption. The base fragment leaders then forward their respective LOE along towards the root $u$ of $T$. Since we have $O(\sqrt{n})$ distinct base fragments, there are at most $O(\sqrt{n})$ LOE edges sent upward in $T$, thus resulting in an additional message complexity of $O(D \sqrt{n})=O(n)$. Taking into account that it takes $O(\sqrt{n})$ rounds for the base fragment leaders to determine the LOE of their fragment, the time complexity amounts to $O(D+\sqrt{n})$.

We now argue the message and time complexity for the case where we execute Phase 3 after Phase 2. Here, we start with $O(n / D)$ distinct fragments each having their own fragment ID and a global BFS tree $T$ of depth $O(D)$. Since each fragment finds one LOE which is first aggregated at the fragment leader and then forwarded along $T$ to the global BFS root, this requires $O(D \cdot n / D)=O(n)$ messages in total and $O(D+n / D)=O(D)$ rounds, since by assumption $D=\Omega(\sqrt{n})$.

Combining the complexity bounds from the previous lemmas we obtain the following theorem:

Theorem 3.14. Consider a synchronous network (in the $K T_{0}$ model) of $n$ nodes, $m$ edges, and diameter $D$, and suppose that at most $O(\log n)$ bits can be transmitted over each link in every round. Algorithm 1 computes an MST and, with high probability, runs in $\tilde{O}(D+\sqrt{n})$ rounds and exchanges $\tilde{O}(m)$ messages.

\section{A SIMULTANEOUSLY TIGHT LOWER BOUND}

As mentioned in Section 1.2, the existing graph construction of [7] and [10] used to establish the lower bound of $\tilde{\Omega}(D+\sqrt{n})$ rounds does not simultaneously yield the message lower bound of $\Omega(m)$; similarly, the existing lower bound graph construction of [31] that shows the message lower bound of $\Omega(m)$ does not simultaneously yield the time lower bound of $\tilde{\Omega}(D+\sqrt{n})$. Previously, Das Sarma et al. [7] presented a sparse graph of $O(n)$ edges to obtain the $\tilde{\Omega}(D+\sqrt{n})$ time bound for 
almost all choices of $D$, while Kutten et al. [31] showed that $\Omega(m)$ messages are required to solve broadcast and hence also for constructing a (minimum) spanning tree. ${ }^{12}$

The following result presents a "universal lower bound" for MST in the sense that it shows that for essentially any $n, m$, and $D$, there exists a class of graphs of $n$ nodes, $m$ edges, and with diameter $D$, such that any randomized MST algorithm takes $\tilde{\Omega}(D+\sqrt{n})$ rounds and $\Omega(m)$ messages on some of those graphs to succeed with constant probability. Our proof combines two lower bound techniques: hardness of distributed symmetry breaking, used to show the lower bound on message complexity [31], and communication complexity, used to show the lower bound on time complexity [7].

THEOREM 4.1. There exists a class of graphs of n nodes, $m$ edges, and diameter $D=\Theta\left(n^{1 / 4}\right),{ }^{13}$ such that any -error distributed MST algorithm for the $K T_{0}$ model requires $\tilde{\Omega}(D+\sqrt{n})$ time and $\Omega(m)$ messages in expectation on some of those graphs, for any sufficiently small constant $\varepsilon>0$. This holds even if nodes have unique IDs (chosen from a range of size poly $(n)$ ), have knowledge of the network size $n$, but do not know the diameter D.

\subsection{Proof of Theorem 4.1}

The Lower Bound Graph. Our lower bound graph $G$ consists of the graph construction $H$ of [46] (and its subsequent refinement in [7]), combined with the dumbbell graph construction of [31]. We first outline the main features of $H$, and refer the reader to [7] for the details. The graph $H$ consists of two designated nodes $s$ and $t$ that are connected by $\Theta(\sqrt{n})$ vertex-disjoint slow paths, each having length $\Theta(\sqrt{n})$ and one highway path of length $D=\Theta\left(n^{1 / 4}\right)$, which determines the diameter of $H$. Consider the nodes on each path as being enumerated starting from 0 . To obtain the required diameter $D=\Theta\left(n^{1 / 4}\right)$, we add spoke edges (i.e., shortcuts) to each node on the highway path: for each $i \in[0, D]$, we connect the $i$ th highway node to the $(i \cdot D)$-th node on each slow path.

We modify the above graph by removing the edge between the two vertices $u_{1}$ and $u_{2}$ on the highway path at distance $\lfloor D / 2\rfloor$ and $\lceil D / 2\rceil+1$ from $s$ and connecting them to one vertex each of a $\left\lceil c_{1} m / n\right\rceil$-regular graph $C$ consisting of $c_{2} n$ nodes, where $c_{1}$ and $c_{2}$ are two positive constants. We assume that $C$ has a strong diameter of $O(\log n)$, where $m \geq c n$, for a sufficiently large positive constant $c .{ }^{14}$ We call the edges of $C$ switch edges. Note that the two vertices of $C$ that are connected to $u_{1}$ and $u_{2}$ have degree $\left\lceil c_{1} m / n\right\rceil+1$.

To obtain a concrete graph from the lower bound construction, we assign unique IDs (chosen from a range of size poly $(n)$ ), and specify a port mapping for each node $u$ that maps $[1, \operatorname{deg}(u)]$ to one of $u$ 's neighbors. We point out that this port mapping function is not known in advance to $u$. For a concrete graph $G$, we define the open graph $G[e]$ as the graph where we have removed edge $e$, and we define $G^{\text {open }}$ to be the set of open graphs obtained by all possible ways of removing any of the switch edges in $C$. After removing an edge $e$ that was connected at port $i$ to node $u$, we call $i$ an open port. Note that this is different from the construction in [31], where $\mathcal{G}^{\text {open }}$ consists of all open graphs considering all possible edge removals. Let $G^{\prime}\left[e^{\prime}\right], G^{\prime \prime}\left[e^{\prime \prime}\right] \in \mathcal{G}^{\text {open }}$ be two open graphs with disjoint node IDs. By connecting the two open ports (due to removing edge $e^{\prime}$ ) of $G^{\prime}$

\footnotetext{
${ }^{12}$ Any algorithm that constructs a spanning tree using $O(f(n))$ messages can be used to elect a leader using $O(f(n)+$ $n$ ) messages in total, by first constructing a spanning tree and then executing any broadcast algorithm restricting its communication to the $O(n)$ spanning tree edges.

${ }^{13}$ Note that this can be strengthened to hold for diameter $\Theta(\log n)$ as described in [7], but here we choose to describe the result for a looser bound for the sake of readability.

${ }^{14}$ Such graphs exist since any random $d$-regular graph is known to be an expander (and hence its diameter is $O(\log n)$ ) with high probability when $d$ is sufficiently large (at least some constant).
} 
to the two open ports in $G^{\prime \prime}$ we obtain the dumbbell graph denoted by Dumbbell $\left(G^{\prime}\left[e^{\prime}\right], G^{\prime \prime}\left[e^{\prime \prime}\right]\right)$. These two new edges are called bridge edges. See Figure 2.

\subsubsection{Part 1: Symmetry Breaking.}

The Complexity of Bridge Crossing and Broadcast. We define the input graph collection $\mathcal{I}$ to be the set of all dumbbell graphs obtained by bridging ID-disjoint open graphs from $\mathcal{G}^{\text {open }}$, which contains all possible 1-edge removals of all possible concrete graphs taking into account all possible port numberings and ID assignments.

The bridge crossing problem was introduced in [31], and captures the hardness of discovering a bridge edge without sending too many messages. In more detail, we say that an algorithm $\mathcal{A}$ solves bridge crossing in an execution $\alpha$ on a graph $I \in \mathcal{I}$, if some vertex sends a message in $\alpha$ across one of the two bridge edges of $I$. We first bound the message complexity of deterministic bridge crossing algorithms on this input class:

LEMMA 4.2. Every deterministic algorithm $\mathcal{A}$ that achieves bridge crossing on at least $1 / 4$ of the dumbbell graphs in the collection $I$ has expected message complexity $\Omega(m)$, if the graph is uniformly sampled from $\mathcal{I}$.

Lemma 4.2 is very similar to Lemma 3.6 in [31], with the difference that our input set $I$ is restricted to the possible dumbbell graph combinations for the switch edges that are in $C$, whereas, in [31], any edge can be a switch edge. Nevertheless, the number of switch edges in $C$ is sufficiently large, i.e.,

$$
|E(C)|=\Theta\left(\frac{m}{2 n}|C|\right)=\Theta(m)=\Theta(|E(G)|),
$$

and hence the same counting argument as in Lemmas 3.5 and 3.6 in [31] can be applied to show an average message complexity of $\Omega(\mathrm{m})$ for solving bridge crossing with a deterministic algorithm when choosing input graphs uniformly from $\mathcal{I}$. As this does not require us to introduce any new technical ideas, we only describe the overall idea of the proof and refer the reader to [31] for the details: The main idea of the proof is to consider Dumbbell $\left(G^{\prime}\left[e^{\prime}\right], G^{\prime \prime}\left[e^{\prime \prime}\right]\right)$, where each of $G^{\prime}$ and $G^{\prime \prime}$ is a copy of $G$ with a concrete port numbering and ID assignment. Let $\mathcal{P}$ be a bridge crossing algorithm and consider the execution of $\mathcal{P}$ on the (disconnected) graph consisting of $G^{\prime}$ and $G^{\prime \prime}$ of $2 n$ nodes. Comparing this with the execution of $\mathcal{P}$ on Dumbbell $\left(G^{\prime}\left[e^{\prime}\right], G^{\prime \prime}\left[e^{\prime \prime}\right]\right)$, an easy indistinguishability argument shows that $\mathcal{P}$ behaves exactly the same in both executions up until the point where bridge crossing happens. In the execution on the disconnected graph, let $t(e)$ be the first time that $\mathcal{P}$ sends a message across $e$, for any $e \in E(C)$, and let $L=\left(e_{1}, \ldots, e_{\ell}\right)$ be a list containing the edges of $G^{\prime}$ in increasing order of $t(e)$, breaking ties in a predetermined way. It follows that, when $\mathcal{P}$ sends the first message across $e_{j}$ in the dumbbell graph $\operatorname{Dumbbell}\left(G^{\prime}\left[e^{\prime}\right], G^{\prime \prime}\left[e^{\prime \prime}\right]\right)$, which occurs at the $j$-th position in $L$, it must have sent at least $j-1$ messages for $e_{1}, \ldots, e_{j-1}$. We obtain the average message complexity for deterministic algorithms by counting the total number of messages in all graphs in $\mathcal{I}$ divided by the number of graphs in the input collection (see Lemma 3.5 in [31]).

Lemma 4.2 can be extended to randomized Monte Carlo algorithms via Yao's Minimax Lemma [50], yielding the following result:

LEMMA 4.3. Let $\mathcal{P}$ be an $\varepsilon$-error randomized bridge crossing algorithm. Then, there exists a graph $G \in \mathcal{I}$ such that the expected message complexity of $\mathcal{P}$ on $G$ is $\Omega(m)$, where the expectation is taken over the random bits of $\mathcal{P}$.

\subsubsection{Part 2: Communication Complexity.}

Reduction from Set Disjointness. The lower bound for MST of [7] is shown by a reduction from the spanning connected subgraph problem, which itself is used in a reduction from the set disjointness 


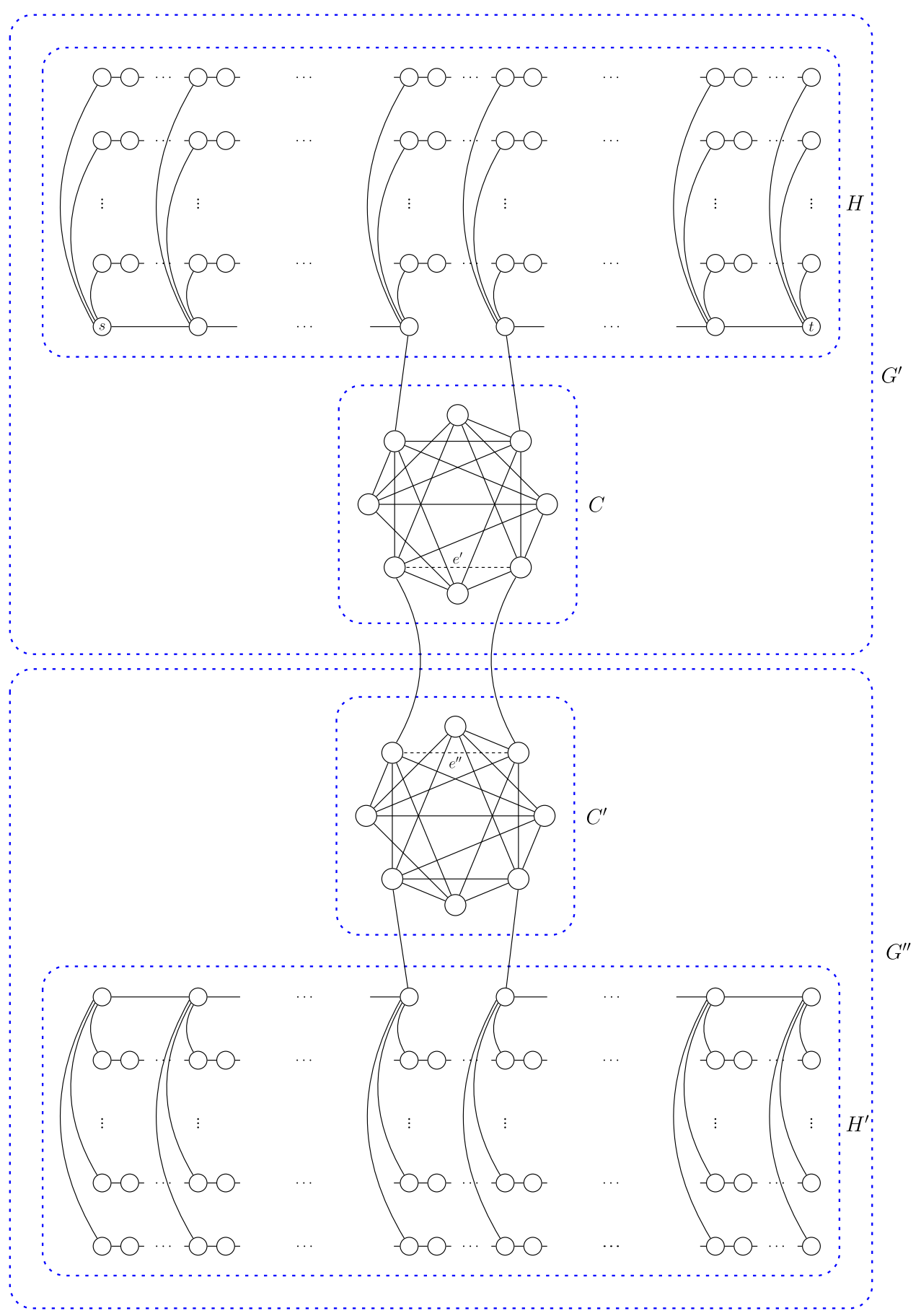

Fig. 2. The graph Dumbbell $\left(G^{\prime}\left[e^{\prime}\right], G^{\prime \prime}\left[e^{\prime \prime}\right]\right)$ for the proof of Theorem 4.1. 
problem in 2-party communication complexity [29]. In the 2-party model, Alice receives $X$ and Bob receives $Y$, for some $b$-bit vectors $X$ and $Y$, and the players communicate along a communication channel to decide if there is an index $i$ such that $X[i]=Y[i]=1$. Razborov [47] showed that any $\varepsilon^{-}$ error randomized communication protocol requires $\Omega(b)$ bits to solve set disjointness. Das Sarma et al. [7] leverage this fact by showing how Alice and Bob can jointly simulate the execution of a distributed MST algorithm $\mathcal{A}$ on a graph with a weight assignment depending on the inputs $X$ and $Y$ to obtain a protocol for set disjointness.

Edge Weight Assignment. All slow path edges and all highway edges obtain weight 1 in $G$, whereas the spoke edges that are not incident to $s$ or $t$ obtain weight $\infty$. We assign weight 1 to all edges in $E(C)$. Recall from the description of the lower bound graph $G$ that there are $\Theta(\sqrt{n})$ slow paths, and hence we set

$$
b=\Theta(\sqrt{n}) .
$$

For every $i \in[1, b]$, the $i$ th spoke edge incident to $s$ is assigned weight 1 if $X[i]=0$, and weight $n$ otherwise. Similarly, the $i$ th spoke edge incident to $t$ is assigned weight 1 if $Y[i]=0$, and weight $n$ otherwise.

Consider, in the MST $M$ of $G$, the $j$ th slow path $\rho_{j}$ connecting $s$ and $t$. A crucial property is that $\rho_{j}$ must contain exactly one spoke incident to either $s$ or $t$ as otherwise $\rho_{j}$ is either disconnected from the rest of the graph or, if both spokes are part of $M$, the highway path forms a cycle with $\rho_{j}$. If $X$ and $Y$ are disjoint, then either the $j$ th spoke incident to Alice has weight 1 or the $j$ th spoke incident to Bob; in this case, the spoke that has weight 1 is part of $M$. Consequently, we have the following result:

Lemma 4.4 (SEe [7]). The MST contains one edge of weight $n$ if and only if $X$ and $Y$ are not disjoint.

Simulating the MST algorithm. Alice and Bob create $G$, assign weights appropriately to the edges incident to $s$ and $t$, and then simulate the execution of $\mathcal{A}$ on $G$. Note that Alice is unaware of Bob's input and hence she does not know the weights of the edges incident to $t$.

Therefore, Alice starts simulating all nodes except $t$ and its neighbors and, similarly, Bob simulates all nodes except $s$ and its neighbors. For completeness, we describe the simulation argument of [7] from Alice's point of view: Alice and Bob need to keep the simulation of nodes $s$ and $t$ afloat, while sending at most $O(\log n)$ bits per simulated round. As mentioned, Alice does not simulate $t$ and its neighbors and hence there are $b+1$ boundary nodes ( $b$ of them are on slow paths and one is on the highway path) among her simulated nodes that may receive messages from nodes that are only simulated by Bob. Let $v$ be Alice's (current) boundary node that is on the highway path. If, in the simulation, $v$ is supposed to receive a message from it's (highway) neighbor $w$ simulated by Bob, then Bob simply sends this message to Alice, requiring $O(\log n)$ bits. On the other hand, Bob does not send any messages to Alice if they concern her boundary nodes on the slow paths. Thus, Alice stops simulating all of its boundary nodes that are on slow paths after the first round. However, for now, she can still simulate the boundary node $v$ on the highway path. We observe that, in each round, Alice loses the ability to simulate one layer of boundary nodes on slow paths, causing their respective neighbors (closer to Alice) to become boundary nodes in the next round. Recall from the lower bound graph construction that $v$ has spoke edges to a set $S$ of $\Theta(\sqrt{n})$ nodes on slow paths. Thus, Alice stops simulating $v$ after the round in which she stops simulating the nodes in $S$, as Bob cannot afford to send all necessary messages that may originate from nodes in $S$ to Alice. In other words, Alice "loses" one of her simulated highway boundary vertices every $\Theta\left(n^{1 / 4}\right)$ rounds. Since the slow paths have length $\Theta(\sqrt{n})$ and there are $\Theta\left(n^{1 / 4}\right)$ many nodes on the highway path, it follows that Alice can continue simulating $s$ for $\Theta(\sqrt{n})$ rounds. 
Once $\mathcal{A}$ terminates, Alice knows which edges incident to $s$ are in the MST and Bob knows the same about $t$. Moreover, since the weight of the MST depends only on these incident edges, Alice can compute the total weight incident to $s$ and then send it to Bob, requiring $O(\log n)$ bits. From this, Bob can reconstruct the total weight of the MST (since all other edges have weight 1). If the MST does not contain any edge of weight $n$, then the total weight is $n-1$ and, by Lemma 4.4, Bob can conclude that $X$ and $Y$ are disjoint. On the other hand, if the MST does contain an edge of weight $n$ (which must be a spoke incident to either $s$ or $t$ ) then there is some index where $X$ and $Y$ intersect. It follows that the solution for MST solves set disjointness and, as described above, each round of the simulation produces at most $O(\log n)$ bits. Consequently, the simulation cannot terminate in $o(b)=o(\sqrt{n} / \log n)$ rounds as this would result in $o(b)$ bits being communicated between Alice and Bob, contradicting the $\Omega(b)$ lower bound for set disjointness [47]. Since this holds for a constant probability of error, an easy application of Markov's inequality shows that the expected time complexity must also be $\tilde{\Omega}(D+\sqrt{n})$ :

Lemma 4.5. There exists a weight function $w$ such that, for any graph $G \in \mathcal{I}$, executing algorithm $\mathcal{A}$ on the weighted graph $G_{w}$, where every edge e has weight $w(e)$, takes $\tilde{\Omega}(D+\sqrt{n})$ rounds in expectation.

4.1.3 Putting Everything Together. We are now ready to combine the results of Lemma 4.3, which we only argued for unweighted graphs so far, with Lemma 4.5. The next lemma directly implies Theorem 4.1.

Lemma 4.6. For any MST algorithm $\mathcal{A}$, there exists a weighted graph $G$ such that $\mathcal{A}$ requires $\tilde{\Omega}(D+\sqrt{n})$ rounds in expectation and has an expected message complexity of $\Omega(m)$.

Proof. Consider an MST algorithm $\mathcal{A}$ and the worst-case weight assignment $w$ provided by Lemma 4.5. Apply $w$ to every graph in the collection $\mathcal{I}$ yielding the collection of weighted graphs $\overline{\mathcal{I}}$. For any $G \in \mathcal{I}$, consider the corresponding weighted graph $\bar{G} \in \overline{\mathcal{I}}$, and let $\bar{C} \subset \bar{G}$ denote the weighted subgraph corresponding to the regular (unweighted) subgraph $C \subset G$ (see Figure 2). According to the edge weight assignment, all edges in $E(C)$ are set to 1 , and these are the only edges that are used as switch edges when constructing the dumbbell graphs required for showing Lemma 4.3. It follows that Lemma 4.3 also applies to the weighted graph collection $\overline{\mathcal{I}}$. Then, by using Markov's inequality to derive probabilities of $2 / 3$ each for both the individual statements in Lemma 4.3 and Lemma 4.5 , the union bound yields that with probability at least $1 / 3$ both the time and the message lower bounds simultaneously hold.

\section{CONCLUSIONS}

We have presented a new distributed algorithm for the fundamental minimum spanning tree problem which is simultaneously time- and message-optimal (to within $\operatorname{polylog}(n)$ factors).

An interesting open question is whether there exists a distributed MST algorithm with nearoptimal time and message complexities in the $K T_{1}$ variant of the model.

Currently, it is not known whether other important problems such as shortest paths, minimum cut, and random walks, enjoy singular optimality. These problems admit distributed algorithms which are (essentially) time-optimal but not message-optimal [8, 24, 37, 38]. Some work in this direction recently started to appear [20,39], but further research is needed to address these questions.

\section{REFERENCES}

[1] Hagit Attiya and Jennifer Welch. 1998. Distributed Computing: Fundamentals, Simulations and Advanced Topics. McGraw-Hill, Inc.

[2] Baruch Awerbuch. 1987. Optimal distributed algorithms for minimum weight spanning tree, counting, leader election, and related problems. In Proceedings of the 19th ACM Symposium on Theory of Computing (STOC). 230-240. 
[3] Baruch Awerbuch, Oded Goldreich, David Peleg, and Ronen Vainish. 1990. A trade-off between information and communication in broadcast protocols. F. ACM 37, 2 (1990), 238-256.

[4] Baruch Awerbuch and David Peleg. 1990. Sparse partitions. In Proceedings of the 31st Annual Symposium on Foundations of Computer Science (FOCS). 503-513.

[5] Francis Chin and H. F. Ting. 1985. An almost linear time and $O(n \log n+e)$ messages distributed algorithm for minimum-weight spanning trees. In Proceedings of the 26th IEEE Symposium on Foundations of Computer Science (FOCS). 257-266.

[6] Richard Cole and Uzi Vishkin. 1986. Deterministic coin tossing and accelerating cascades: micro and macro techniques for designing parallel algorithms. In Proceedings of the 18th Annual ACM Symposium on Theory of Computing (STOC). 206-219.

[7] Atish Das Sarma, Stephan Holzer, Liah Kor, Amos Korman, Danupon Nanongkai, Gopal Pandurangan, David Peleg, and Roger Wattenhofer. 2012. Distributed verification and hardness of distributed approximation. SIAM f. Comput. 41, 5 (2012), 1235-1265.

[8] Atish Das Sarma, Danupon Nanongkai, Gopal Pandurangan, and Prasad Tetali. 2013. Distributed random walks. f. ACM 60, 1, Article 2 (2013).

[9] Michael Elkin. 2006. A faster distributed protocol for constructing a minimum spanning tree. f. Comput. Syst. Sci. 72 , 8 (2006), 1282-1308.

[10] Michael Elkin. 2006. An unconditional lower bound on the time-approximation trade-off for the distributed minimum spanning tree problem. SIAM f. Comput. 36, 2 (2006), 433-456.

[11] Michael Elkin. 2017. A simple deterministic distributed MST algorithm, with near-optimal time and message complexities. In Proceedings of the 2017 ACM Symposium on Principles of Distributed Computing (PODC). 157-163.

[12] Michalis Faloutsos and Mart Molle. 2004. A linear-time optimal-message distributed algorithm for minimum spanning trees. Distributed Computing 17, 2 (2004), 151-170.

[13] Eli Gafni. 1985. Improvements in the time complexity of two message-optimal election algorithms. In Proceedings of the 4th Symposium on Principles of Distributed Computing (PODC). 175-185.

[14] Robert G. Gallager, Pierre A. Humblet, and Philip M. Spira. 1983. A distributed algorithm for minimum-weight spanning trees. ACM Trans. Program. Lang. Syst. 5, 1 (1983), 66-77.

[15] Juan A. Garay, Shay Kutten, and David Peleg. 1998. A sublinear time distributed algorithm for minimum-weight spanning trees. SIAM f. Comput. 27, 1 (1998), 302-316.

[16] Mohsen Ghaffari and Bernhard Haeupler. 2016. Distributed algorithms for planar networks II: Low-congestion shortcuts, MST, and min-cut. In Proceedings of the 27th Annual ACM-SIAM Symposium on Discrete Algorithms (SODA) 202-219.

[17] Mohsen Ghaffari and Fabian Kuhn. 2018. Distributed MST and broadcast with fewer messages, and faster gossiping. In Proceedings of the 32nd International Symposium on Distributed Computing (DISC). 30:1-30:12.

[18] Mohsen Ghaffari, Fabian Kuhn, and Hsin-Hao Su. 2017. Distributed MST and routing in almost mixing time. In Proceedings of the 2017 ACM Symposium on Principles of Distributed Computing (PODC). 131-140.

[19] Robert Gmyr and Gopal Pandurangan. 2018. Time-message trade-offs in distributed algorithms. In Proceedings of the 32nd International Symposium on Distributed Computing (DISC). 32:1-32:18.

[20] Bernhard Haeupler, D. Ellis Hershkowitz, and David Wajc. 2018. Round- and message-optimal distributed graph algorithms. In Proceedings of the 2018 ACM Symposium on Principles of Distributed Computing (PODC). 119-128.

[21] Bernhard Haeupler, Taisuke Izumi, and Goran Zuzic. 2016. Low-congestion shortcuts without embedding. In Proceedings of the 2016 ACM Symposium on Principles of Distributed Computing (PODC). 451-460.

[22] Bernhard Haeupler, Taisuke Izumi, and Goran Zuzic. 2016. Near-optimal low-congestion shortcuts on bounded parameter graphs. In Proceedings of the 30th International Symposium on Distributed Computing (DISC). 158-172.

[23] James W. Hegeman, Gopal Pandurangan, Sriram V. Pemmaraju, Vivek B. Sardeshmukh, and Michele Scquizzato. 2015. Toward optimal bounds in the congested clique: Graph connectivity and MST. In Proceedings of the 2015 ACM Symposium on Principles of Distributed Computing (PODC). 91-100.

[24] Monika Henzinger, Sebastian Krinninger, and Danupon Nanongkai. 2016. A deterministic almost-tight distributed algorithm for approximating single-source shortest paths. In Proceedings of the 48th ACM Symposium on Theory of Computing (STOC). 489-498.

[25] Maleq Khan and Gopal Pandurangan. 2008. A fast distributed approximation algorithm for minimum spanning trees. Distributed Computing 20, 6 (2008), 391-402.

[26] Valerie King, Shay Kutten, and Mikkel Thorup. 2015. Construction and impromptu repair of an MST in a distributed network with $o(m)$ communication. In Proceedings of the 2015 ACM Symposium on Principles of Distributed Computing (PODC). 71-80.

[27] Hartmut Klauck, Danupon Nanongkai, Gopal Pandurangan, and Peter Robinson. 2015. Distributed computation of large-scale graph problems. In Proceedings of the 26th Annual ACM-SIAM Symposium on Discrete Algorithms (SODA). 391-410. 
[28] Liah Kor, Amos Korman, and David Peleg. 2013. Tight bounds for distributed minimum-weight spanning tree verification. Theory Comput. Syst. 53, 2 (2013), 318-340.

[29] Eyal Kushilevitz and Noam Nisan. 1997. Communication Complexity. Cambridge University Press.

[30] Shay Kutten, Danupon Nanongkai, Gopal Pandurangan, and Peter Robinson. 2014. Distributed symmetry breaking in hypergraphs. In Proceedings of the 28th International Symposium on Distributed Computing (DISC). 469-483.

[31] Shay Kutten, Gopal Pandurangan, David Peleg, Peter Robinson, and Amitabh Trehan. 2015. On the complexity of universal leader election. 7. ACM 62, 1, Article 7 (2015).

[32] Shay Kutten and David Peleg. 1998. Fast distributed construction of small $k$-dominating sets and applications. f. Algorithms 28, 1 (1998), 40-66.

[33] Christoph Lenzen. 2016. Lecture Notes on Theory of Distributed Systems. https://www.mpi-inf.mpg.de/fileadmin/inf/ d1/teaching/winter15/tods/ToDS.pdf.

[34] Christoph Lenzen and Boaz Patt-Shamir. 2014. Improved distributed steiner forest construction. In Proceedings of the 2014 ACM Symposium on Principles of Distributed Computing (PODC). 262-271.

[35] Nancy Lynch. 1996. Distributed Algorithms. Morgan Kaufmann Publishers.

[36] Ali Mashreghi and Valerie King. 2017. Time-communication trade-offs for minimum spanning tree construction. In Proceedings of the 18th International Conference on Distributed Computing and Networking (ICDCN). Article 8.

[37] Danupon Nanongkai. 2014. Distributed approximation algorithms for weighted shortest paths. In Proceedings of the 46th ACM Symposium on Theory of Computing (STOC). 565-573.

[38] Danupon Nanongkai, Atish Das Sarma, and Gopal Pandurangan. 2011. A tight unconditional lower bound on distributed randomwalk computation. In Proceedings of the 30th Annual ACM Symposium on Principles of Distributed Computing (PODC). 257-266.

[39] Shreyas Pai, Gopal Pandurangan, Sriram V. Pemmaraju, Talal Riaz, and Peter Robinson. 2017. Symmetry breaking in the Congest model: Time- and message-efficient algorithms for ruling sets. In Proceedings of the 31st International Symposium on Distributed Computing (DISC). 38:1-38:16.

[40] Gopal Pandurangan. 2019. Distributed Network Algorithms. https://sites.google.com/site/gopalpandurangan/dna.

[41] Gopal Pandurangan, David Peleg, and Michele Scquizzato. 2016. Message lower bounds via efficient network synchronization. In Proceedings of the 23rd International Colloquium on Structural Information and Communication Complexity (SIROCCO). 75-91.

[42] Gopal Pandurangan, Peter Robinson, and Michele Scquizzato. 2017. A time- and message-optimal distributed algorithm for minimum spanning trees. In Proceedings of the 49th Annual ACM Symposium on the Theory of Computing (STOC). 743-756.

[43] Gopal Pandurangan, Peter Robinson, and Michele Scquizzato. 2018. The distributed minimum spanning tree problem. Bulletin of the EATCS 125 (2018).

[44] David Peleg. 1998. Distributed matroid basis completion via elimination upcast and distributed correction of minimum-weight spanning trees. In Proceedings of the 25th International Colloquium on Automata, Languages and Programming (ICALP). 164-175.

[45] David Peleg. 2000. Distributed Computing: A Locality-Sensitive Approach. Society for Industrial and Applied Mathematics.

[46] David Peleg and Vitaly Rubinovich. 2000. A near-tight lower bound on the time complexity of distributed minimumweight spanning tree construction. SIAM f. Comput. 30, 5 (2000), 1427-1442.

[47] Alexander A. Razborov. 1992. On the distributional complexity of disjointness. Theor. Comput. Sci. 106, 2 (1992), 385390.

[48] Robert Endre Tarjan. 1983. Data Structures and Network Algorithms. Society for Industrial and Applied Mathematics.

[49] Gerard Tel. 1994. Introduction to Distributed Algorithms. Cambridge University Press.

[50] Andrew Chi-Chih Yao. 1977. Probabilistic computations: Toward a unified measure of complexity. In Proceedings of the 18th Annual Symposium on Foundations of Computer Science (FOCS). 222-227.

Received March 2018; revised August 2019; accepted September 2019 